\title{
Reactions of 1,3-Dithiolane 1,3-Dioxides with Nucleophiles
}

\author{
Wen-Chih Chou, Sheng-Ann Yang and Jim-Min Fang* \\ Department of Chemistry, National Taiwan University, Taipei, Taiwan 10617, Republic of China
}

\begin{abstract}
Reaction of benzenethiol with the 1,3-dithiolane 1,3-dioxides 3a-f gave the 1,4-dithiane 1 -oxides 4-5, the $\alpha, \beta$-unsaturated sulfoxides 6-9, the ring-opening products 10-11 and various reduction products. Addition of benzenethiol, malononitrile, diethyl malonate or 2-mercapto-4,5-dihydrothiazole to the double bonds of the 2-alkenyl-1,3-dithiolane 1,3-dioxides $3 \mathbf{g}-\mathbf{h}$ was performed with methylmagnesium chloride in methanol. Addition of methanol or an allyl group to the dioxide $3 \mathrm{~h}$ occurred regioselectively to give the 1,3-dithiolane 1 -oxide 19 or the 1,3-dithiolane 20.
\end{abstract}

Sulfoxides are versatile reagents in organic synthesis, ${ }^{1}$ being used as acyl nucleophile equivalents, in dehydrosulfinylation to give alkenes, in the Pummerer rearrangement to give carbonyl compounds and in [2,3] sigmatropic rearrangements to give allylic alcohols. Sulfoxides are intrinsically chiral reagents and their use in asymmetric control is well documented. ${ }^{2}$ In contrast, there has been little work reported with disulfoxides, ${ }^{3}$ although compounds such as the trans-1,3-dithiolane 1,3dioxides, which have $C_{2}$-symmetry, are potential auxiliaries for enantioselective reactions. For example, Aggarwal and coworkers have shown both stereoselective addition reactions of trans-1,3-dithiane 1,3-dioxides with aromatic aldehydes and Diels-Alder reactions of trans-2-methylene-1,3-benzodithiole 1,3-dioxide with dienes. Khiar and his co-workers have demonstrated the use of $C_{2}$-symmetric disulfoxides as chiral ligands in asymmetric Diels-Alder reactions. We report herein the addition and ring-expansion of 1,3-dithiolane 1,3-dioxides 3a-h in the presence of benzenethiol and other nucleophiles.

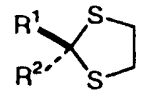

$1 a-h$<smiles>[R7]C1([R7])SCC[S+]1[O-]</smiles>

$2 a-h$<smiles>[R7]C1([R7])[S+]([O-])CC[S+]1[O-]</smiles>

$3 a-h$

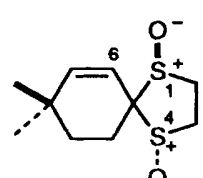

$\mathbf{3 b}$

\section{Results and Discussion}

Preparation of Disulfoxides 3a-h.-Although 1,3-dithiolanes can be oxidized directly to their corresponding 1,3-dioxides by use of 2 equiv. of $\mathrm{NaIO}_{4},{ }^{4}$ we carried out this transformation in two successive oxidation steps, as shown in Table 1. This method provided an ambiguous assignment of the stereostructures of the disulfoxides $\mathbf{3 a}-\mathbf{h}$. Oxidation of the 1,3-dithiolanes 1a-h with 1 equiv. of $\mathrm{NaIO}_{4}$ gave the corresponding monosulfoxdes $\mathbf{2 a - h}$ in favour of the trans-isomers. ${ }^{5}$ The dithiolane 1d which had 2-methyl and 2-ethoxycarbonyl substituents afforded only the trans-isomer $\mathbf{2 d}$, where the sulfinyl oxygen and ethoxycarbonyl group were on opposite faces of the molecule. ${ }^{6}$ In the ${ }^{1} \mathrm{H}$ NMR spectra of compounds 2 , the $2-\mathrm{H}$ or $2-\mathrm{Me}$ resonances of the trans-isomers usually occurred at lower field than those of the $c i s$-isomers. ${ }^{7}$ The monosulfoxides $\mathbf{2 a - h}$ were then oxidized with 1 equiv. of $m$-chloroperbenzoic acid $(m-$ CPBA ${ }^{8}$ to give the corresponding disulfoxides $3 \mathbf{a}-\mathbf{h}$. The presence of $\mathrm{C}=\mathrm{C}$ double bonds did not interfere with the oxidation process. Since the trans- and cis-isomers of $\mathbf{2 b}$ were both converted into the disulfoxide $\mathbf{3 b}$, the product must have the trans-configuration. Similarly, the disulfoxides $\mathbf{3 d - h}$ must also have the trans-configuration. The disulfoxides $\mathbf{3 a}$ and $\mathbf{3 c}$ exist as a mixture of two isomers, where the $c i s$-isomers have the two oxygen atoms and the methyl group on the same face to
Table 1 Conversion of the dithiolanes $1 \mathbf{a}-\mathbf{h}$ to the disulfoxides $3 \mathbf{a}-\mathbf{h}$ via the monosulfoxides $\mathbf{2 a - h}$

\begin{tabular}{|c|c|c|}
\hline Dithiolane $\mathrm{R}^{1}, \mathrm{R}^{2}$ & $\begin{array}{l}\text { Monosulfoxide }{ }^{a} \\
\text { (yield \%; ratio of } \\
\text { trans }: \text { cis isomers) }\end{array}$ & $\begin{array}{l}\text { Disulfoxide }{ }^{b} \\
\text { (yield } \% \text {; ratio of } \\
\text { trans: cis isomers) }\end{array}$ \\
\hline $\begin{array}{l}\text { 1a } \mathrm{Ph}, \mathrm{Me} \\
\text { 1b } \mathrm{CH}=\mathrm{CHCMe}_{2} \mathrm{CH}_{2} \mathrm{CH}_{2} \\
\text { 1c } \mathrm{Me}\left[\mathrm{CH}_{2}\right]_{4}, \mathrm{Me} \\
\text { 1d } \mathrm{EtO}_{2} \mathrm{C}, \mathrm{Me} \\
\text { 1e } \mathrm{MeCH}_{2} \mathrm{CH}_{2}, \mathrm{H} \\
\text { 1f } \mathrm{PhCH}=\mathrm{CH}, \mathrm{Me} \\
\text { 1g } \mathrm{PhCH}=\mathrm{CH}, \mathrm{H} \\
\text { 1h } \mathrm{MeCH}=\mathrm{CH}, \mathrm{H}\end{array}$ & $\begin{array}{l}\text { 2a }(66 ; 84: 16) \\
\text { 2b }(54 ; 59: 41) \\
\text { 2c }(76 ; 66: 34) \\
\text { 2d }(42 ; 100: 0) \\
2 \text { e }(37 ; 72: 28) \\
\text { 2f }(24 ; 63: 27) \\
2 \mathrm{~g}(86 ; 75: 25) \\
2 \mathrm{~h}(80 ; 75: 25)\end{array}$ & $\begin{array}{l}\text { 3a }(64 ; 25: 75)^{c} \\
\text { 3b }(80 ; 100: 0) \\
\text { 3c }(87 ; 80: 20)^{c} \\
\text { 3d }(84 ; 100: 0) \\
\text { 3e }(44 ; 100: 0) \\
\text { 3f }(60 ; 100: 0) \\
\text { 3g }(55 ; 100: 0) \\
\text { 3h }(100 ; 100: 0)\end{array}$ \\
\hline
\end{tabular}

${ }^{a}$ Oxidant, $\mathrm{NaIO}_{4}$ ( 1 equiv.). ${ }^{b}$ Oxidant $m$-CPBA ( 1 equiv.). ${ }^{c}$ The cisisomer of $3 \mathbf{a}$ (or $3 \mathbf{c}$ ) had the two oxygen atoms and the methyl group on the same face.

form a $C_{\mathrm{s}}$-symmetric molecule, as inferred by the coalescence of the $\mathrm{C}-4$ and $\mathrm{C}-5$ resonances. These results indicate that oxidation of the 1,3-dithiolane 1-oxides $\mathbf{2 b}$ and $2 \mathbf{d}-\mathbf{h}$ occurred exclusively on the face opposite to the sulfinyl oxygen, whereas oxidation of the 1,3-dithiolane 1-oxides $2 \mathbf{a}$ and $\mathbf{2 c}$ were less selective, owing to the steric effect of the phenyl or the pentyl group.

Thermal Reactions of the Disulfoxides 3a-e in the Presence of Benzenethiol. - A methanolic solution of dioxide 3a and benzenethiol ( 1 equiv.) was heated at reflux to give the dithiane monooxide 4 in $72 \%$, as a mixture of the trans- and cis-isomers $(86: 14)$. The $2-\mathrm{H}$ and $\mathrm{C}-2$ resonances in the trans isomer of 4 appeared at low field ( $\delta 3.97$ and 68.7), compared to the signals for the cis isomer $(\delta 3.85$ and 61.3$)$. The $\mathrm{C}-5$ resonance occurs at $\delta 25.5$ for the trans isomer, indicating an equatorial sulfinyl oxygen, whereas it resonates at $\delta 16.1$ for the cis isomer, indicating an axial configuration. ${ }^{9}$ Thermal ring expansion of the 2-methyl-1,3-dithiolane 1-oxide ${ }^{10}$ has been shown to involve an $\mathrm{H}$-shift from the methyl group to the sulfinyl oxygen, accompanied by a concurrent ring opening to give an intermediate vinylthioethylsulfenic acid. The product 5,6-dihydro-1,4-dithiine is subsequently obtained by a ringclosure process presumably via the sulfur-stabilized carbonium ion intermediate. Accordingly, ring-expansion of the dioxide 3a to compound 4 was probably initiated by formation of the sulfenic acid intermediate $\mathbf{A}$ shown in Scheme 1. However, the intermediate $\mathrm{A}$ containing an electron deficient $\mathrm{C}=\mathrm{C}$ double bond should act differently from the electrophilic vinyl sulfide species described above. The sulfenic acid $\mathbf{A}$ might undergo an intramolecular Michael addition ${ }^{11}$ to give a disulfoxide $\mathbf{B}$, in which the less hindered sulfoxide group is selectively reduced. 


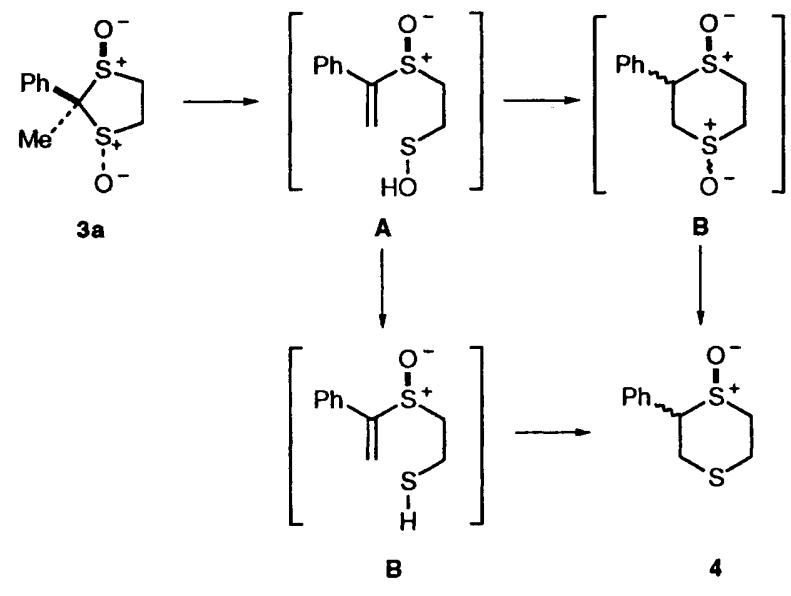

Scheme 1 Reagents and conditions: $\mathrm{PhSH}, \mathrm{MeOH}, 70^{\circ} \mathrm{C}, 36 \mathrm{~h}(72 \%)$<smiles>CC1(C)CC([O-])C2(C=CC(C)(C)SCC[Sn]2[O-])C1</smiles>

5

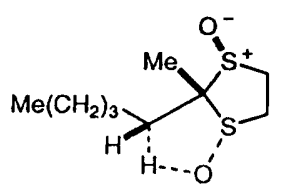

c<smiles>C=C(CC)[S+]([O-])CC[Al]</smiles>

$6 \mathrm{Y}=\mathrm{H}$
$\mathbf{Y}=\mathrm{SPh}$<smiles>CCOC(C)(C)[S+]([O-])CCS(OC)(Sc1ccccc1)S(C)(C)C</smiles>

D

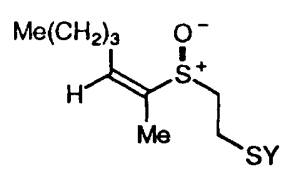

$\mathrm{Y}=\mathrm{H}$
$\mathrm{Y} Y=\mathrm{SPh}$<smiles>CCOC(=O)C([N+](=O)[O-])[S+]([O-])CC[Al]</smiles>

$10 Y=H$ $11 \mathrm{Y}=\mathrm{SPh}$
Alternatively, reduction of the sulfenic acid $\mathbf{A}$ to the thiol $\mathbf{B}^{\prime}$, followed by an intramolecular Michael addition would lead to the same product. The 1,3-dithiolane 1,3-dioxide $3 \mathbf{b}$, generated from 4,4-dimethylcyclohex-2-enone, underwent a similar ring expansion to afford the 1,4-dithiane 1 -oxide $5(73 \%)$. Since the ring-junction protons $(1-\mathrm{H}$ and $6-\mathrm{H})$ in compound 5 showed a small coupling constant $(3.2 \mathrm{~Hz})$, this bicyclic compound was determined to have a cis fused ring-junction and the resonance of $\mathrm{C}-4$ at $\delta 21.7$ was taken to indicate an equatorial sulfinyl oxygen. ${ }^{9}$ The reaction of 2-methyl-2-pentyl-1,3-dithiolane 1,3 dioxide $3 \mathbf{c}$ under similar conditions gave the $a, \beta$-unsaturated sulfoxides 6 and 8 accompanied by a significant amount of the disulfide 7. This reaction was considered to involve the sulfenic acid intermediates generated from deprotonation of either the methyl or the pentyl group, similar to that shown in Scheme 1. Subsequent disproportionation of these sulfenic acid intermediates with benzenethiol would lead to the observed products. From the distribution of products, $(6+7): 8=7: 1$, deprotonation of the methyl group appeared to be the more favoured process. In order to promote the ring closure of compounds 6 and 8 , the reaction temperature was raised to $110^{\circ} \mathrm{C}$ for a prolonged period. However, such conditions yielded mainly the disulfides 7 and 9 , but no cyclization product. A $16 \%$ nuclear Overhauser enhancement of the vinyl proton $(\delta 6.18)$ in the sulfoxide 8 was observed by irradiation of the adjacent methyl group ( $\delta 1.83$ ), indicating the $Z$-configuration for compound 8 . Due to the deshielding effect of the sulfoxide group, the vinyl proton on the $\beta$-carbon of $E$-isomer usually resonates at lower field (about $\delta 6.4$ ) than that of the $Z$-isomer. ${ }^{12}$ The configuration of 9 was assigned as $Z$-, due to the vinyl resonance at $\delta 6.12$ which was close to the value observed for compound $\mathbf{8}$. The stereochemical outcome was in agreement with a concerted $\mathrm{H}$-shift as shown in $\mathbf{C}$.

Treatment of the dithiolane dioxide $3 \mathrm{~d}$ with benzenethiol in refluxing methanol gave the thiol $\mathbf{1 0}$ and the corresponding phenyl disulfide 11. These products were characterised by characteristic methyl doublets in the ${ }^{1} \mathrm{H}$ NMR spectra. The reaction sequence was presumably initiated by direct attack ${ }^{13}$ of benzenethiol on one of the sulfoxide groups to give the intermediate anion $\mathbf{D}$, stabilized by the other adjacent ester and the other sulfoxide group.

2-Propyl-1,3-dithiolane 1,3-dioxide $3 \mathrm{e}$ was heated to $110^{\circ} \mathrm{C}$ in a sealed tube in the presence of benzenethiol to give the partially reduced product 2 e. Partial deoxygenation of the disulfoxide $3 f$ gave the monosulfoxide $2 \mathrm{f}$ on treatment with lithium ethanethiolate ( 1 equiv.) and complete deoxygenation gave the dithiolane 1 f on treatment with lithium dimethylcuprate ( 2 equiv.). These results were consistent with a previous report on the reduction of vinyl sulfoxides with EtMgBr-CuI. ${ }^{12}$

Addition to the $\mathrm{C}=\mathrm{C}$ Double Bond of the Disulfoxides $3 \mathrm{~g}$ and 3h.--Allyl sulfoxides are known to undergo [2,3] sigmatropic rearrangements to form intermediates, which can be reduced with benzenethiol to give allyl alcohols. ${ }^{14}$ However, it was noted that benzenethiol adds to the $\mathrm{C}=\mathrm{C}$ double bond of 2styryl-1,3-dithiolane 1,3-dioxide $3 \mathrm{~g}$ in $\mathrm{MeOH}$ upon mediation with a Grignard reagent to give the disulfoxide 12 in $91 \%$ yield. Use of malonotrile as the nucleophile gave the addition product 13 as a diastereoisomeric mixture $(67: 33)$. Similar reactions of 2-prop-1-enyl-1,3-dithiolane 1,3-dioxide 3 h with the nucleophiles benzenethiol, malononitrile, diethyl malonate and 2mercapto-4,5-dihydrothiazole in the presence of $\mathrm{MeMgCl}$ gave the addition products $14,15,16$ and 17 respectively. In one case, addition of malononitrile to the disulfoxide $3 \mathrm{~h}$ was mediated by $\mathrm{Al}_{2} \mathrm{O}_{3} \cdot{ }^{15}$<smiles>[Y]C(c1ccccc1)C([R])C1[S+]([O-])CC[S+]1[O-]</smiles>

$12 \mathrm{R}=\mathrm{SPh}, \mathrm{Y}=\mathrm{H}$

$\left[{ }^{2} \mathrm{H}\right]-12 \mathrm{R}=\mathrm{SPh}, \mathrm{Y}=\mathrm{D}$

$13 \mathrm{R}=\mathrm{CH}(\mathrm{CN})_{2}, \mathrm{Y}=\mathrm{H}$

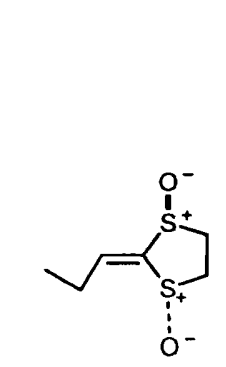

18

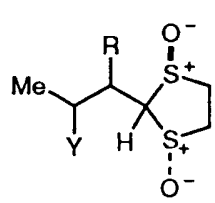

$14 R=S P h, Y=H$

$\left.I^{2} \mathrm{H}\right]-14 \mathrm{R}=\mathrm{SPh}, \mathrm{Y}=\mathrm{D}$

$\begin{array}{rl}15 & R=C H(C N)_{2}, Y=H \\ \left.{ }^{2} H\right]-15 & R=C H(C N)_{2}, Y=D\end{array}$ $16 \mathrm{R}=\mathrm{CH}\left(\mathrm{CO}_{2} \mathrm{Et}\right)_{2}, \mathrm{Y}=\mathrm{H}$<smiles></smiles><smiles>CCC=C1SCC[Se]1[O-]</smiles>

$18 \mathrm{a}$
Two possible mechanisms were considered to account for the observed additions (Scheme 2). Path a evoked direct addition of the nucleophile to the $\mathrm{C}=\mathrm{C}$ double bond; the intermediate might be stabilised by coordination to the sulfinyl oxygen as shown in E. Path $b$ involved a primary isomerisation of compound $\mathbf{3 g}$ (or $3 h$ ) to the conjugated disulfoxide $\mathbf{F}$, which then underwent a Michael-type reaction ${ }^{16}$ with the nucleophile to give the observed product. Path a seemed less likely since the addition of EtSH to 2-methyl-2-styryl-1,3-dithiolane 1,3-dioxide 3f, containing no hydrogen atom at C-2 failed (Table 2, entry 7). We thought the addition performed in MeOD would afford doubly deuteriated products $\mathbf{G}$ via path $b$. To our surprise, the monodeuteriated addition product $\left[{ }^{2} \mathrm{H}\right]-12$ was obtained from 
Table 2 Reactions of 1,3-dithiolane 1,3-dioxides 3a-h

\begin{tabular}{|c|c|c|c|}
\hline Entry & Disulfoxide & Reaction conditions & $\begin{array}{l}\text { Products (yield, \%; ratio of } \\
\text { isomers) }\end{array}$ \\
\hline 1 & 3a & $\mathrm{PhSH}, \mathrm{MeOH}, 70^{\circ} \mathrm{C}, 36 \mathrm{~h}$ & $4(72 ; 86: 14)$ \\
\hline 2 & 3b & $\mathrm{PhSH}, \mathrm{MeOH}, 70^{\circ} \mathrm{C}, 22 \mathrm{~h}$ & $5(73)$ \\
\hline 3 & 3c & $\mathrm{PhSH}, \mathrm{MeOH}, 70^{\circ} \mathrm{C}, 40 \mathrm{~h}$ & $6(10)+7(10)+8(3)$ \\
\hline 4 & 3c & $\mathrm{PhSH}, \mathrm{MeOH}, 110^{\circ} \mathrm{C}$ (sealed), $12 \mathrm{~h}$ & $7(22)+9(10)$ \\
\hline 5 & 3d & $\mathrm{PhSH}, \mathrm{MeOH}, 70^{\circ} \mathrm{C}, 3 \mathrm{~h}$ & $10(14 ; 80: 20)+11(41 ; 67: 33)$ \\
\hline 6 & $3 \mathbf{e}$ & $\mathrm{PhSH}, \mathrm{MeOH}, 110^{\circ} \mathrm{C}$ (sealed), $18 \mathrm{~h}$ & $2 e(27)+3 e(50)$ \\
\hline 7 & $3 f$ & EtSH, BuLi, THF, room temp., $4 \mathrm{~h}$ & $2 f(43)$ \\
\hline 8 & $3 f$ & $\mathrm{Me}_{2} \mathrm{CuLi}, \mathrm{Et}_{2} \mathrm{O}, \mathrm{THF}, 0^{\circ} \mathrm{C}, 6 \mathrm{~h}$ & 1f (36) \\
\hline 9 & $3 \mathrm{~g}$ & $\mathrm{PhSH}, \mathrm{MeMgCl}, \mathrm{MeOH}$, room temp., $24 \mathrm{~h}$ & $12(91 ; 50: 50)$ \\
\hline 10 & 3g & PhSH, MeMgCl, MeOD, room temp., $24 \mathrm{~h}$ & {$\left[{ }^{2} \mathrm{H}\right]-12(92)^{a}$} \\
\hline 11 & 3g & $\mathrm{CH}_{2}(\mathrm{CN})_{2}, \mathrm{MeMgCl}, \mathrm{MeOH}$, room temp., $19 \mathrm{~h}$ & $13(42 ; 67: 33)$ \\
\hline 12 & $\mathbf{3 h}$ & $\mathrm{PhSH}, \mathrm{MeMgCl}, \mathrm{MeOH}$, room temp., $24 \mathrm{~h}$ & $14(98 ; 57: 43)$ \\
\hline 13 & 3h & $\mathrm{PhSH}, \mathrm{MeMgCl}, \mathrm{MeOD}$, room temp., $24 \mathrm{~h}$ & {$\left[{ }^{2} \mathrm{H}\right]-14(100 ; 62: 38)$} \\
\hline 14 & $3 \mathbf{h}$ & $\mathrm{CH}_{2}(\mathrm{CN})_{2}, \mathrm{MeMgCl}, \mathrm{MeOH}$, room temp., $1 \mathrm{~h}$ & $15(46 ; 67: 33)$ \\
\hline 15 & $\mathbf{3 h}$ & $\mathrm{CH}_{2}(\mathrm{CN})_{2}, \mathrm{MeMgCl}, \mathrm{MeOD}$, room temp., $1 \mathrm{~h}$ & {$\left[{ }^{2} \mathrm{H}\right]-15(11)^{a, b}$} \\
\hline 16 & $\mathbf{3 h}$ & $\mathrm{CH}_{2}(\mathrm{CN})_{2}, \mathrm{Al}_{2} \mathrm{O}_{3}$, room temp., $72 \mathrm{~h}$ & $15(28 ; 56: 44)^{b}$ \\
\hline 17 & 3h & $\mathrm{CH}_{2}\left(\mathrm{CO}_{2} \mathrm{Et}\right)_{2}, \mathrm{MeMgCl}, \mathrm{MeOH}$, room temp., $1 \mathrm{~h}$ & $16(46 ; 71: 29):$ \\
\hline 18 & 3h & 2-mercapto-4,5-dihydro-thiazole, $\mathrm{MeMgCl}, \mathrm{MeOH}$, room temp., $0.5 \mathrm{~h}$ & $17(83 ; 100: 0)$ \\
\hline 19 & $\mathbf{3 h}$ & $\mathrm{MeCO}_{2} \mathrm{H}, \mathrm{MeMgCl}, \mathrm{MeOH}$, room temp., $1 \mathrm{~h}$ & $19(55 ; 33: 33: 17: 17)$ \\
\hline 20 & $\mathbf{3 h}$ & $\mathrm{CH}_{2}=\mathrm{CHCH}_{2} \mathrm{SiMe}_{3},\left(\mathrm{CF}_{3} \mathrm{CO}\right)_{2} \mathrm{O}$, THF, room temp., $12 \mathrm{~h}$ & $20(53)$ \\
\hline
\end{tabular}

${ }^{a}$ Ratio of isomers was not determined. ${ }^{b}$ Starting material $3 \mathrm{~h}$ was recovered.

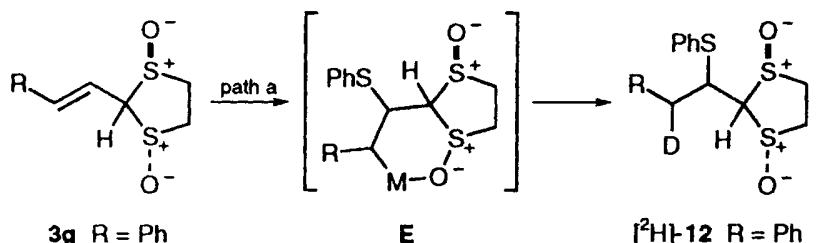
$\begin{array}{ll}3 \mathrm{~g} R=\mathrm{Me} & \mathrm{E} \\ {\left[{ }^{2} \mathrm{H}\right]-12 \mathrm{~A}=\mathrm{Me}}\end{array}$<smiles>CC1C[C@@H]1C</smiles>

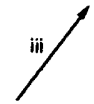<smiles>[2H]C(C)/C=C1\[Si](=CC)CC[S+]1[O-]</smiles>

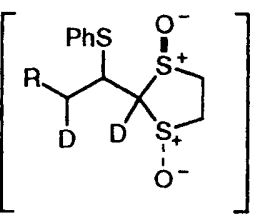

G

Scheme 2 Reagents and conditions: $\mathrm{PhSH}, \mathrm{MeMgCl}, \mathrm{MeOD}$, room temp. 24 h. Path a: direct addition. Path b: i, isomerisation; ii, Michael addition; iii, work-up with aq. $\mathrm{NH}_{4} \mathrm{Cl}$.

the reaction of the disulfoxide $\mathbf{3 g}$ with benzenethiolate ion in MeOD. Similarly, the monodeuteriated product $\left[{ }^{2} \mathrm{H}\right]-14$ or $\left[{ }^{2} \mathrm{H}\right]-15$ was also obtained from the reaction of the sulfoxide $3 \mathrm{~h}$ with either benzenethiol or malononitrile with the Grignard $\mathrm{MeMgCl}$ in $\mathrm{MeOD}$. Since there was no deuterium atom incorporated at $\mathrm{C}-2$ in the final product, we assumed a rapid exchange of the $\mathrm{C}_{2}-\mathrm{D}$, even in the presence of aqueous $\mathrm{NH}_{4} \mathrm{Cl}$. From literature, ${ }^{4}$ the acidity of $2-\mathrm{H}$ in 1,3-dithiane 1,3dioxide has been measured to have a $p K_{a}$ value of 25.0 . To support our assumption, the addition of the conjugated disulfoxide 18 with $\mathrm{PhSMgCl}$ in $\mathrm{MeOD}$, followed by work-up with aqueous $\mathrm{NH}_{4} \mathrm{Cl}$, gave the adduct 14 containing no deuterium atom.

Treatment of 2-prop-1-enyl-1,3-dithiolane 1,3-dioxide 3h with chloromagnesium acetate in $\mathrm{MeOH}$ gave 2-(2-methoxypropylidene)-1,3-dithiolane 1-oxide 19 as a mixture of four diastereoisomers $(\mathbf{a}: \mathbf{b}: \mathbf{c}: \mathbf{d}=33: 33: 17: 17)$. In the ${ }^{1} \mathrm{H}$ NMR spectrum, the vinyl resonances of the major $Z$-isomers $(\mathbf{a}+\mathbf{b})$ occurred at $\delta 5.94$ and 5.99 , respectively, whereas the minor
$E$-isomers (c and d) resonated at lower field $(\delta 6.40$ and 6.44 respectively). The reaction presumably proceeded with an intermediate $\alpha, \beta$-unsaturated dithiolanium ion $\mathbf{H}$ (Scheme 3 ) derived from a Pummerer rearrangement similar to that proposed for the reactions of sulfoxides with Grignard reagents or allyltrimethylsilane. ${ }^{17}$ Efficient trapping with methanol of the intermediate $\mathbf{H}$, which presumably adopts the favourable trans-conformation at $\mathrm{C}\left(1^{\prime}\right)-\mathrm{C}(2)$, leads to the formation of the $Z$-isomers. Similarly, the reaction of the dioxide $3 \mathrm{~h}$ with allyltrimethylsilane in the presence of trifluoroacetic anhydride, gave the diallylated product 20 . In both reactions, the nucleophile added selectively at the $\gamma$-carbon $\left(C-2^{\prime}\right)$ of the $\alpha, \beta$ unsaturated dithiolanium intermediate. The second allyl group was introduced by a subsequent Michael-type reaction to give the intermediate $\mathbf{I}$, which then underwent isomerisation to furnish the final product $\mathbf{2 0}$.

\section{Experimental}

General information concerning instrumentation and materials has been described previously. ${ }^{18} J$ Values are given in $\mathbf{H z}$.

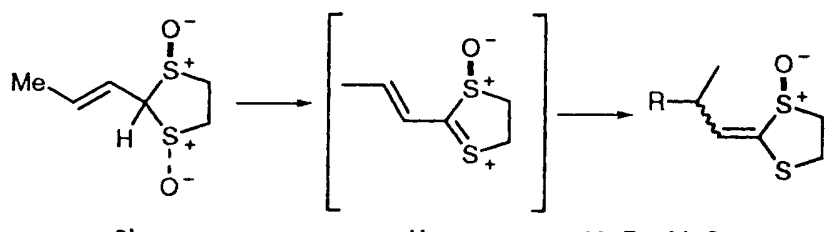

3h

H $\begin{aligned} 19 \mathrm{R} & =\mathrm{MeO} \\ 20 \mathrm{a} \mathrm{R} & =\mathrm{CH}_{2}=\mathrm{CHCH}_{2}\end{aligned}$<smiles>C=CCC(=C1SCCS1)C(C)CC=C</smiles>

20 I

Scheme 3 Reagents and conditions: $\mathrm{MeCO}_{2} \mathrm{H}, \mathrm{MeMgCl}, \mathrm{MeOH}$, room temp., $1 \mathrm{~h}$, giving $19(55 \%)$, or $\mathrm{CH}_{2}=\mathrm{CHCH}_{2} \mathrm{SiMe}_{3},\left(\mathrm{CF}_{3} \mathrm{CO}_{2}\right)_{2} \mathrm{O}$, THF, room temp., $12 \mathrm{~h}$, giving $20(53 \%)$ 
1,3-Dithiolanes 1a-h were prepared from the corresponding carbonyl compounds by literature methods. ${ }^{19}$

General Procedure for Oxidation of the Dithiolanes 1a-h to the Disulfoxides 3a-h via the Monosulfoxides $\mathbf{2 a - h}$.-A solution of 2-methyl-2-phenyl-1,3-dithiolane $1 \mathrm{a}(1.26 \mathrm{~g}, 6.1 \mathrm{mmol})$ in $\mathrm{MeOH}\left(70 \mathrm{~cm}^{3}\right)$ was stirred with aqueous $\mathrm{NaIO}_{4}(1.3 \mathrm{~g}, 6.1$ mmol in $15 \mathrm{~cm}^{3}$ water) at $0^{\circ} \mathrm{C}$ for $12 \mathrm{~h}$. The yellow solid was filtered off and the filtrate was concentrated and then extracted with EtOAc $\left(3 \times 30 \mathrm{~cm}^{3}\right)$. The extracts were combined, washed with brine $\left(3 \times 30 \mathrm{~cm}^{3}\right)$, dried $\left(\mathrm{Na}_{2} \mathrm{SO}_{4}\right)$ and filtered. The filtrate was concentrated and chromatographed on silica gel with EtOAc-hexane ( $1: 1)$ to give the monosulfoxide $2 \mathrm{a}(0.85 \mathrm{~g}, 66 \%)$ as a mixture of the trans- and cis-isomers (84:16). The monosulfoxide $2 \mathrm{a}$ in $\mathrm{CH}_{2} \mathrm{Cl}_{2}\left(40 \mathrm{~cm}^{3}\right)$ was treated with a solution of $m$-CPBA $(0.68 \mathrm{~g}, 4 \mathrm{mmol})$ in $\mathrm{CH}_{2} \mathrm{Cl}_{2}\left(10 \mathrm{~cm}^{3}\right)$ at $0{ }^{\circ} \mathrm{C}$ for $2 \mathrm{~h}$. The white solid was filtered off and the filtrate washed with aqueous $\mathrm{NaOH}(20 \%)$, dried $\left(\mathrm{Na}_{2} \mathrm{SO}_{4}\right)$, concentrated and the residue chromatographed on silica gel with EtOAc to give the disulfoxide $3 \mathrm{a}(0.58 \mathrm{~g}, 64 \%)$ as a white solid. This was obtained as a mixture of the trans- and cisisomers $(25: 75)$, when the cis-sulfoxides are also cis to the methyl group.

2-Methyl-2-phenyl-1,3-dithiolane 1-oxide 2a. A mixture of trans- and cis-isomers $(66 \% ; 84: 16)$; oil: $\left[R_{\mathrm{f}} 0.49\right.$, hexaneEtOAc (1:4)]; $m / z 212\left(\mathrm{M}^{+}, 78 \%\right), 136(68)$ and $121(100)$ $\delta_{\mathrm{H}}\left(\mathrm{CDCl}_{3}\right) 1.92(\mathrm{~s}, \mathrm{Me}$ cis $), 1.98(\mathrm{~s}, \mathrm{Me}$ trans), 2.63-2.79 $(1 \mathrm{H}$, $\mathrm{m}), 3.02-3.14(1 \mathrm{H}, \mathrm{m}$, dithiolane ring $\mathrm{H}), 3.21-3.33(1 \mathrm{H}, \mathrm{m})$, 3.61-3.75 (1 H, m), 7.21-7.38 (3 H, m) and 7.58-7.64 (2 H, m); $\delta_{\mathrm{C}}\left(\mathrm{CDCl}_{3}\right) 23.0$ (q, Me trans) $/ 25.0$ (q, Me cis), $31.9(\mathrm{t}, \mathrm{C}-4)$, $52.6(\mathrm{t}, \mathrm{C}-5$ trans $) / 52.0(\mathrm{t}), 82.9(\mathrm{~s}, \mathrm{C}-2$ trans $) / 84.0(\mathrm{~s}), 127.35$ (d, 2 C), 128.1 (s), 128.4 (d) and 128.4 (d, 2 C) (Found: $\mathrm{M}^{+}$, 212.0313. Calc. for $\mathrm{C}_{10} \mathrm{H}_{12} \mathrm{OS}_{2}: M, 212.0330$ ).

8,8-Dimethyl-1,4-dithiaspiro[4.5]dec-6-ene 1-oxide $\mathbf{2 b}$. Two diastereoisomers $(54 \%$; $59: 41)$ were separated by HPLC on a $\mu$-Porsasil column with EtOAc as eluent. Major isomer: oil; HPLC(EtOAc) $t_{\mathrm{r}} 7.7 \mathrm{~min} ; v_{\max }$ (neat) $/ \mathrm{cm}^{-1} 1054 ; \mathrm{m} / z 216\left(\mathrm{M}^{+}\right.$, $79 \%), 199(38)$ and $140(100) ; \delta_{\mathrm{H}}\left(\mathrm{CDCl}_{3}\right) 1.04(3 \mathrm{H}, \mathrm{s}), 1.05(3$ $\mathrm{H}, \mathrm{s}), 1.68-1.76(2 \mathrm{H}, \mathrm{m}), 1.94-2.08(1 \mathrm{H}, \mathrm{m}), 2.44-2.54(1 \mathrm{H}$, $\mathrm{m}), 3.10-3.22(1 \mathrm{H}, \mathrm{m}), 3.28-3.41(2 \mathrm{H}, \mathrm{m}), 3.62-3.76(1 \mathrm{H}$, $\mathrm{m}), 5.27\left(1 \mathrm{H}, \mathrm{d}, J\right.$ 9.9) and $5.69(1 \mathrm{H}, \mathrm{d}, J 9.9) ; \delta_{\mathrm{C}}\left(\mathrm{CDCl}_{3}\right)$ $24.3(\mathrm{t}), 28.5(\mathrm{q}), 29.3(1), 30.3(\mathrm{t}), 31.7(\mathrm{~s}), 35.5(\mathrm{t}), 52.8(\mathrm{t})$, $77.0(\mathrm{~s}), 123.3$ (d) and 143.5 (d) (Found: $\mathrm{M}^{+}, 216.0636$. Calc. for $\mathrm{C}_{10} \mathrm{H}_{16} \mathrm{OS}_{2}: M, 216.0642$ ). Minor isomer: white solid, m.p. $90-91{ }^{\circ} \mathrm{C}$; HPLC (EtOAc) $t_{\mathrm{r}} 9.5 \mathrm{~min} ; v_{\max }(\mathrm{KBr}) / \mathrm{cm}^{-1}$ $1054 ; m / z 216\left(\mathrm{M}^{+}, 75 \%\right), 199(42)$ and $140(100) ; \delta_{\mathrm{H}}\left(\mathrm{CDCl}_{3}\right)$ $1.03(3 \mathrm{H}, \mathrm{s}), 1.04(3 \mathrm{H}, \mathrm{s}), 1.64-2.05(4 \mathrm{H}, \mathrm{m}), 3.05-3.21(1$ $\mathrm{H}, \mathrm{m}), 3.33-3.49(2 \mathrm{H}, \mathrm{m}), 3.80-3.94(1 \mathrm{H}, \mathrm{m}), 5.73(1 \mathrm{H}, \mathrm{d}, J$ $10.0, \mathrm{H}-\mathrm{b})$ and $5.88(1 \mathrm{H}, \mathrm{d}, J 10.0) ; \delta_{\mathrm{C}}\left(\mathrm{CDCl}_{3}\right) 28.3(\mathrm{q}), 29.1$ $(\mathrm{q}), 31.35(\mathrm{~s}), 31.65(\mathrm{t}), 32.7(\mathrm{t}), 34.3(\mathrm{t}), 54.8(\mathrm{t}), 76.4(\mathrm{~s})$ 119.5 (d) and 144.0 (d) (Found: $\mathrm{M}^{+}, 216.0642$. Calc. for $\left.\mathrm{C}_{10} \mathrm{H}_{16} \mathrm{OS}_{2}: M, 216.0642\right)$.

2-Methyl-2-pentyl-1,3-dithiolane 1-oxide 2c. A mixture of trans- and cis-isomers (76\%; 66:34); oil; TLC (hexane-EtOAc, $1: 4) R_{\mathrm{f}} 0.34 ; v_{\max }($ neat $) / \mathrm{cm}^{-1} 1449$ and $1052 ; \mathrm{m} / \mathrm{z} 206\left(\mathrm{M}^{+}, 76 \%\right)$ and $55(100) ; \delta_{\mathrm{H}}\left(\mathrm{CDCl}_{3}\right) 0.80(3 \mathrm{H}, \mathrm{t}, J 6.9), 1.20-1.29(4 \mathrm{H}$, $\mathrm{m}), 1.40$ (s, Me cis), 1.55 (s, Me trans), 1.35-1.59 and 1.70 $1.95(4 \mathrm{H}, \mathrm{m}), 3.07-3.29(3 \mathrm{H}, \mathrm{m})$ and $3.45-3.64(1 \mathrm{H}, \mathrm{m})$; $\delta_{\mathrm{C}}\left(\mathrm{CDCl}_{3}\right) 13.75(\mathrm{q}), 19.0\left(\mathrm{q}, \mathrm{C}-1^{\prime \prime}\right.$ trans $) / 24.3(\mathrm{q}), 22.1(\mathrm{t}) / 22.2$ $(\mathrm{t}), 24.95(\mathrm{t}) / 25.95(\mathrm{t}), 29.6(\mathrm{t}) / 30.9(\mathrm{t}), 31.7(\mathrm{t}) / 31.8(\mathrm{t}), 38.8$ $(\mathrm{t}) / 34.3(\mathrm{t}), 54.1(\mathrm{t}) / 53.6(\mathrm{t})$ and $75.6(\mathrm{~s}) / 77.1(\mathrm{~s})$ (Found: $\mathrm{M}^{+}$, 206.0811. Calc. for $\mathrm{C}_{9} \mathrm{H}_{18} \mathrm{OS}_{2}: M, 206.0799$ ).

2-Ethoxycarbonyl-2-methyl-1,3-dithiolane 1-oxide 2d. transIsomer: oil $(42 \%)$ TLC (EtOAc) $R_{\mathrm{r}} 0.49 ; v_{\max }\left(\right.$ neat) $/ \mathrm{cm}^{-1}$ 1722,1251 and $1052 ; m / z 209\left(\mathrm{M}^{+}+1,41 \%\right)$ and $59(100)$; $\delta_{\mathrm{H}}\left(\mathrm{CDCl}_{3}\right) 1.31(3 \mathrm{H}, \mathrm{t}, J 7.1), 1.80(3 \mathrm{H}, \mathrm{s}), 3.39-3.46(2 \mathrm{H}, \mathrm{m})$, 3.55-3.66 (1 H, m), 3.79-3.87 (1 H, m) and 4.22 (2 H, q, J 7.1); $\delta_{\mathrm{C}}\left(\mathrm{CDCl}_{3}\right) 13.9(\mathrm{q}), 17.6(\mathrm{q}), 34.6(\mathrm{t}), 53.3(\mathrm{t}), 62.6(\mathrm{t}), 79.5(\mathrm{~s})$ and 170.4 (s) (Found: $\mathrm{M}^{+}, 208.0230$. Calc. for $\mathrm{C}_{7} \mathrm{H}_{12} \mathrm{O}_{3} \mathrm{~S}_{2}, M$, 208.0228).

2-Propyl-1,3-dithiolane 1-oxide 2e. A mixture of trans- and cis-isomers $(37 \%$; $72: 28)$ : oil; TLC (hexane-EtOAc, $1: 4) R_{\mathrm{f}} 0.20$; $v_{\max }($ neat $) / \mathrm{cm}^{-1} 1458$ and $1039 ; \mathrm{m} / \mathrm{z} 165\left(\mathrm{M}^{+}+1,100 \%\right)$ and $164\left(\mathrm{M}^{+}, 97\right) ; \delta_{\mathrm{H}}\left(\mathrm{CDCl}_{3}\right) 0.85(\mathrm{t}, J 7.3) / 0.89(\mathrm{t}, J$ 7.3), 1.34 $2.18(4 \mathrm{H}, \mathrm{m}), 2.66-2.81(1 \mathrm{H}, \mathrm{m}), 3.19-3.34(2 \mathrm{H}, \mathrm{m}), 3.48-3.60$ $(1 \mathrm{H}, \mathrm{m})$ and $4.02(\mathrm{t}, J 7.3,2-\mathrm{H}$ trans $) / 3.98(\mathrm{t}, J 7.3) ; \delta_{\mathrm{C}}\left(\mathrm{CDCl}_{3}\right)$ 13.3(q)/13.6(q), 21.2(t)/22.2(t), 31.0(t)/29.7(t), 34.9(t)/30.7(t), $54.3(\mathrm{t}) / 55.7(\mathrm{t})$ and $73.1(\mathrm{~d}) / 68.3(\mathrm{~d})$ (Found: $\mathrm{M}^{+}, 164.0333$. Calc. for $\mathrm{C}_{6} \mathrm{H}_{12} \mathrm{OS}_{2}: M, 164.0330$ )

2-Methyl-2-(2-phenylvinyl)-1,3-dithiolane 1-oxide 2f. A mixture of trans- and cis-isomer $(24 \% ; 63: 27)$ : TLC (EtOAc-hexane, 1:1) $R_{\mathrm{f}} 0.2 ; v_{\max }(\mathrm{KBr}) / \mathrm{cm}^{-1} 2967,1598,1445,1100$ and 1053 ; $m / z 238\left(\mathrm{M}^{+}, 73 \%\right)$ and $161(100) ; \delta_{\mathrm{H}}\left(\mathrm{CDCl}_{3}\right) 1.87(\mathrm{~s}, \mathrm{Me}$ trans) $/ 1.78(\mathrm{~s}), 3.14-3.86(4 \mathrm{H}, \mathrm{m}), 6.24(1 \mathrm{H}, \mathrm{d}, J 16) / 6.53(\mathrm{~d}, J$ 16), $6.88(1 \mathrm{H}, \mathrm{d}, J 15.6) / 6.85(\mathrm{~d}, J 16)$ and $7.29-7.48(5 \mathrm{H}, \mathrm{m})$; $\delta_{\mathrm{C}}\left(\mathrm{CDCl}_{3}\right) 14.0(\mathrm{q}) / 23.5(\mathrm{q}), 32.0(\mathrm{t}) / 29.0(\mathrm{t}), 53.2(\mathrm{t}) / 52.9(\mathrm{t})$, 79.2 (s)/74.1 (s), 126.6 (d, 2 C)/127.6 (d), 126.7 (d)/124.1 (d), 128.3 (d), 128.5 (d, 2 C), 134.8 (d)/133.4 (d) and 135.2 (s)/135.6 (s) (Found: $\mathrm{M}^{+}, 238.0480$. Calc. for $\mathrm{C}_{12} \mathrm{H}_{14} \mathrm{OS}_{2}: M$, 238.0486).

2-(2-Phenylvinyl)-1,3-dithiolane 1-oxide $2 \mathrm{~g}$. A mixture of trans- and cis-isomers $(86 \% ; 75: 25)$ : oily solid, TLC (EtOAchexane, 1:1) $R_{\mathrm{f}} 0.16 ; v_{\max }($ neat $) / \mathrm{cm}^{-1} 2931,1635,1446,1309$ and $1049 ; m / z 224\left(\mathrm{M}^{+}, 11 \%\right)$ and $115(100) ; \delta_{\mathrm{H}}\left(\mathrm{CDCl}_{3}\right) 2.84$ $3.95(4 \mathrm{H}, \mathrm{m}), 5.03(\mathrm{~d}, J 8,2-\mathrm{H}) / 4.96(\mathrm{~d}, J 9), 6.05(\mathrm{dd}, J 14$ and 8 , $1-\mathrm{H}) / 6.33$ (dd, $J 16$ and 9), $6.84\left(\mathrm{~d}, J 14,2^{\prime}-\mathrm{H}\right) / 6.98(\mathrm{~d}, J 16)$ and 7.29-7.46 $(5 \mathrm{H}, \mathrm{m}) ; \delta_{\mathrm{C}}\left(\mathrm{CDCl}_{3}\right) 31.9(\mathrm{t}, \mathrm{C}-4) / 32.2(\mathrm{t}), 53.7(\mathrm{t}$, C-5)/56.4 (t), 75.2 (d, C-2)/70.1 (d), $121.5\left(\mathrm{~d}, \mathrm{C}-1^{\prime}\right) / 119.0$ (d), 126.8 (d)/126.9 (d), 128.5 (d), 128.6 (d), 128.7 (d), 135.5 (s)/135.6 (s) and $136.4\left(\mathrm{~d}, \mathrm{C}-2^{\prime}\right) / 136.7$ (d) (Found: $\mathrm{M}^{+}, 224.0323$. Calc. for $\left.\mathrm{C}_{11} \mathrm{H}_{12} \mathrm{OS}_{2}: M, 224.0339\right)$.

2-Prop-1-enyl-1,3-dithiolane 1-oxide $\mathbf{2 h}$. trans-Isomer: colourless crystals $(80 \%)$, m.p. $108-109^{\circ} \mathrm{C}$, HPLC (EtOAc-hexane, 4:1) $t_{\mathrm{r}} 7.2 \mathrm{~min} ; v_{\max }(\mathrm{KBr}) / \mathrm{cm}^{-1} 2993,1655,1035$ and $926 ; \mathrm{m} / z$ $\left(\mathrm{M}^{+}, 9 \%\right), 147(5), 129(8)$ and $108(100) ; \delta_{\mathrm{H}}\left(\mathrm{CDCl}_{3}\right) 1.70(3 \mathrm{H}$, d, $J 6, \mathrm{Me}), 2.68-3.02(4 \mathrm{H}, \mathrm{m}), 4.75(\mathrm{~d}, J 9,2-\mathrm{H}), 5.32$ (dd, $J 15$ and $\left.9,1^{\prime}-\mathrm{H}\right)$ and $6.04\left(\mathrm{dq}, J 15\right.$ and $\left.6,2^{\prime}-\mathrm{H}\right)$ (Found: $\mathrm{M}^{+}$ 162.0182. Calc. for $\left.\mathrm{C}_{6} \mathrm{H}_{10} \mathrm{OS}_{2}: M, 162.0173\right)$. Cis-isomer: liquid, HPLC (EtOAc-hexane, $4: 1) t_{\mathrm{r}} 8.4 \mathrm{~min} ; v_{\max }\left(\right.$ neat) $/ \mathrm{cm}^{-1}$ $2912,1656,1423,1036$ and $926 ; \delta_{\mathrm{H}}\left(\mathrm{CDCl}_{3}\right) 1.75(\mathrm{~d}, J 6, \mathrm{Me})$, $2.80-3.90(4 \mathrm{H}, \mathrm{m}), 4.70(\mathrm{~d}, J 9, \mathrm{H}-2), 5.50\left(\mathrm{dd}, J 15\right.$ and $\left.9,1^{\prime}-\mathrm{H}\right)$ and $5.90\left(\mathrm{dq}, J 6\right.$ and $\left.15,2^{\prime}-\mathrm{H}\right)$.

2-Methyl-2-phenyl-1,3-dithiolane 1,3-dioxide 3a. A mixture of trans- and cis-isomers $(64 \% ; 25: 75)$ inseparable by chromatography, m.p. $116-118^{\circ} \mathrm{C}$; TLC (MeOH-EtOAc, 1:9) $R_{\mathrm{f}} 0.25$; $v_{\max }(\mathrm{KBr}) / \mathrm{cm}^{-1} 1591,1057$ and $694 ; m / z 229\left(\mathrm{M}^{+}+1,3 \%\right)$, $108(92)$ and $84(100) ; \delta_{\mathrm{H}}\left(\mathrm{CDCl}_{3}\right) 1.77(\mathrm{~s}, \mathrm{Me}$ trans)/1.95 (s, $\mathrm{Me}$ cis $)$, 3.46-3.72 $(4 \mathrm{H}, \mathrm{m})$ and 7.16-7.38 $(5 \mathrm{H}, \mathrm{m}) ; \delta_{\mathrm{C}^{-}}$ $\left(\mathrm{CDCl}_{3}\right) 13.8(\mathrm{q}) / 15.8(\mathrm{q}), 50.05(\mathrm{t}, \mathrm{C}-4$ and $\mathrm{C}-5, \mathrm{cis}), 50.9(\mathrm{t}$, C-5, trans), 53.9 (t, C-4, trans), 86.3 (s, C-2, cis), 91.1 (s, C-2, trans), 126.9 (d), 128.0 (d), 128.6 (d), 129.0 (d), 129.35 (d) and 135.35 (s), (Found: $\mathrm{M}^{+}, 228.0270$. Calc. for $\mathrm{C}_{10} \mathrm{H}_{12} \mathrm{O}_{2} \mathrm{~S}_{2}: M$, 228.0279).

8,8-Dimethyl-1,4-dithiaspiro[4.5]dec-6-ene 1,4-dioxide $\mathbf{3 b}$. White solid (80\%), m.p. $131-132{ }^{\circ} \mathrm{C}$; TLC (EtOAc) $R_{\mathrm{f}} 0.25$; $v_{\max }(\mathrm{KBr}) / \mathrm{cm}^{-1} 1043 ; \mathrm{m} / \mathrm{z} 232\left(\mathrm{M}^{+}, 5 \%\right)$ and $125(100)$; $\delta_{\mathrm{H}}\left(\mathrm{CDCl}_{3}\right) 1.09(3 \mathrm{H}, \mathrm{s}, \mathrm{Me}), 1.10(3 \mathrm{H}, \mathrm{s}, \mathrm{Me}), 1.67-1.73(2 \mathrm{H}$, $\mathrm{m}), 1.81-1.95(1 \mathrm{H}, \mathrm{m}), 2.28-2.42(1 \mathrm{H}, \mathrm{m}), 3.55-3.82(4 \mathrm{H}, \mathrm{m})$, $5.43(1 \mathrm{H}, \mathrm{d}, J 10.1)$ and $6.09(1 \mathrm{H}, \mathrm{d}, J 10.1) ; \delta_{\mathrm{C}}\left(\mathrm{CDCl}_{3}\right) 20.6$ (t), $28.4(\mathrm{q}), 28.7(\mathrm{q}), 31.6(\mathrm{~s}), 34.8(\mathrm{t}), 50.9(\mathrm{t}), 51.9(\mathrm{t}), 88.5(\mathrm{~s})$, 114.2 (d) and 146.8 (d) (Found: $\mathrm{M}^{+}, 232.0597$. Calc. for $\left.\mathrm{C}_{10} \mathrm{H}_{16} \mathrm{O}_{2} \mathrm{~S}_{2}: M, 232.0592\right)$

2-Methyl-2-pentyl-1,3-dithiolane 1,3-dioxide 3c. A mixture of trans- and cis-isomers $(87 \% ; 80: 20)$ were separated by chromatography on silica gel (MeOH-EtOAc, 1:9). transIsomer: white solid, m.p. $56-57^{\circ} \mathrm{C}$; TLC (MeOH-EtOAc, 1:9) 
$R_{\mathrm{f}} 0.30 ; v_{\max }(\mathrm{KBr}) / \mathrm{cm}^{-1} 1460$ and $1035 ; m / z 223\left(\mathrm{M}^{+}+1\right.$, $27 \%)$ and $108(100) ; \delta_{\mathrm{H}}\left(\mathrm{CDCl}_{3}\right) 0.87(3 \mathrm{H}, \mathrm{t}, J 6.8), 1.22-1.40$ $(4 \mathrm{H}, \mathrm{m}), 1.38(3 \mathrm{H}, \mathrm{s}), 1.46-2.00(4 \mathrm{H}, \mathrm{m})$ and $3.44-3.81(4 \mathrm{H}, \mathrm{m})$; $\delta_{\mathrm{C}}\left(\mathrm{CDCl}_{3}\right) 13.8(\mathrm{q}), 13.9(\mathrm{q}), 22.3(\mathrm{t}), 25.3(\mathrm{t}), 30.75(\mathrm{t}), 31.9(\mathrm{t})$, $49.95(\mathrm{t}), 51.0(\mathrm{t})$ and $90.0(\mathrm{~s})$ (Found: $\mathrm{M}^{+}, 222.0766$. Calc. for $\left.\mathrm{C}_{9} \mathrm{H}_{18} \mathrm{O}_{2} \mathrm{~S}_{2}: M, 222.0748\right)$. cis-Isomer: white solid, m.p. $60-$ $61{ }^{\circ} \mathrm{C}$; TLC (MeOH-EtOAc, $\left.1: 9\right) R_{\mathrm{f}} 0.24 ; v_{\max }(\mathrm{KBr}) / \mathrm{cm}^{-1} 1438$ and 1035; $m / z 223\left(\mathrm{M}^{+}+1,15 \%\right), 206(40)$ and $108(100)$; $\delta_{\mathrm{H}}\left(\mathrm{CDCl}_{3}\right) 0.80(3 \mathrm{H}, \mathrm{t}, J 6.7), 1.17-1.28(4 \mathrm{H}, \mathrm{m}), 1.51(3 \mathrm{H}, \mathrm{s})$, $1.40-1.53(4 \mathrm{H}, \mathrm{m})$ and 3.34-3.64 $(4 \mathrm{H}, \mathrm{m}) ; \delta_{\mathrm{C}}\left(\mathrm{CDCl}_{3}\right) 11.3(\mathrm{q})$, $13.6(\mathrm{q}), 22.0(\mathrm{t}), 24.3(\mathrm{t}), 31.6(\mathrm{t}), 34.0(\mathrm{t}), 48.6(\mathrm{t}, 2 \mathrm{C})$ and 82.7 (s) (Found: $\mathrm{M}^{+}, 222.0738$. Calc. for $\mathrm{C}_{9} \mathrm{H}_{18} \mathrm{O}_{2} \mathrm{~S}_{2}: M, 222.0748$ ). 2-Ethoxycarbonyl-2-methyl-1,3-dithiolane 1,3-dioxide 3d. White solid (84\%), m.p. $91-92^{\circ} \mathrm{C}$; TLC (EtOAc) $R_{\mathrm{f}} 0.27$; $v_{\max }(\mathrm{KBr}) / \mathrm{cm}^{-1} 1733,1246$ and $1045 ; \mathrm{m} / \mathrm{z} 225\left(\mathrm{M}^{+}+1\right.$, $100 \%)$ and $211(43) ; \delta_{\mathrm{H}}\left(\mathrm{CDCl}_{3}\right) 1.32(3 \mathrm{H}, \mathrm{t}, J 7.2), 1.75(3 \mathrm{H}$, s), 3.63-3.84 (3 H, m), 3.99-4.16(1 H, m) and $4.29(2 \mathrm{H}, \mathrm{q}, J 7.2)$; $\delta_{\mathrm{C}}\left(\mathrm{CDCl}_{3}\right) 12.7(\mathrm{q}), 13.9(\mathrm{q}), 51.6(\mathrm{t}), 53.3(\mathrm{t}), 63.1(\mathrm{t}), 94.1(\mathrm{~s})$ and 163.9 (s) (Found: $\mathrm{M}^{+}, 224.0176$. Calc. for $\mathrm{C}_{7} \mathrm{H}_{12} \mathrm{O}_{4} \mathrm{~S}_{2}: M$, 224.0177).

2-Propyl-1,3-dithiolane 1,3-dioxide 3e. White solid (44\%), m.p. $95-96{ }^{\circ} \mathrm{C}$; TLC (MeOH-EtOAc, $\left.1: 9\right) R_{\mathrm{f}} 0.23 ; v_{\max }(\mathrm{KBr}) / \mathrm{cm}^{-1}$ 1455 and $1021 ; m / z 180\left(\mathrm{M}^{+}, 100 \%\right) ; \delta_{\mathrm{H}}\left(\mathrm{CDCl}_{3}\right) 1.07(\mathrm{t}, J 7.2$, Me), $1.73(2 \mathrm{H}$, sexet, J 7.2), 1.94-2.06 (2 H, m), 3.57-3.80 (4 H, $\mathrm{m})$ and $3.84(1 \mathrm{H}, \mathrm{d}, J 7) ; \delta_{\mathrm{C}}\left(\mathrm{CDCl}_{3}\right) 13.7(\mathrm{t}), 22.0(\mathrm{t}), 25.4(\mathrm{t})$, $50.7(\mathrm{t}), 51.8(\mathrm{t})$ and 91.0 (d) (Found: $\mathrm{M}^{+}, 180.0274$. Calc. for $\left.\mathrm{C}_{6} \mathrm{H}_{12} \mathrm{O}_{2} \mathrm{~S}_{2}: M, 180.0278\right)$.

2-Methyl-2-(2-phenylvinyl)-1,3-dithiolane 1,3-dioxide $3 \mathbf{f}$. White solid $(60 \%)$, m.p. $109-110^{\circ} \mathrm{C}$; TLC (EtOAc) $R_{\mathrm{f}} 0.17$; $v_{\max }(\mathrm{KBr}) / \mathrm{cm}^{-1} 1636,1492,1400,1089$ and $1039 ; \mathrm{m} / \mathrm{z} 254\left(\mathrm{M}^{+}\right.$, $7 \%)$ and $129(100) ; \delta_{\mathrm{H}}\left(\mathrm{CDCl}_{3}\right) 1.67(3 \mathrm{H}, \mathrm{s}), 3.46-3.86(4 \mathrm{H}$, $\mathrm{m}), 6.35\left(\mathrm{~d}, J 16,1^{\prime}-\mathrm{H}\right), 6.75\left(\mathrm{~d}, J 16,2^{\prime}-\mathrm{H}\right)$ and $7.30-7.43(5 \mathrm{H}$, $\mathrm{m}) ; \delta_{\mathrm{C}}\left(\mathrm{CDCl}_{3}\right) 13.8(\mathrm{q}, 2 \mathrm{C}), 50.5(\mathrm{t}), 51.3(\mathrm{t}), 88.2(\mathrm{~s}), 118.9$ (d), $126.3(\mathrm{~d}, 2 \mathrm{C}), 128.1$ (d, 2 C), $128.2(\mathrm{~d}), 134.8(\mathrm{~d})$ and 134.9 (s) (Found: $\mathrm{M}^{+}, 254.0436$. Calc. for $\mathrm{C}_{12} \mathrm{H}_{14} \mathrm{O}_{2} \mathrm{~S}_{2}: M, 254.0435$ ).

2-(2-Phenylvinyl)-1,3-dithiolane 1,3-dioxide 3g. White crystal (55\%), m.p. $155-157^{\circ} \mathrm{C}$ (from EtOAc); TLC (EtOAc) $\boldsymbol{R}_{\mathrm{f}} \mathbf{0 . 1 7}$; $v_{\max }(\mathrm{KBr}) / \mathrm{cm}^{-1}$ 2927, 1662, 1389, 1102 and $1031 ; \mathrm{m} / \mathrm{z} 240$ $\left(\mathrm{M}^{+}, 14 \%\right)$ and $147(100) ; \delta_{\mathrm{H}}\left(\mathrm{CDCl}_{3}\right) 3.68-3.92(4 \mathrm{H}, \mathrm{m}), 4.72$ $(\mathrm{d}, J 10,2-\mathrm{H}), 6.15\left(\mathrm{dd}, J 16\right.$ and $\left.10,1^{\prime}-\mathrm{H}\right), 6.98\left(\mathrm{~d}, J 16,2^{\prime}-\mathrm{H}\right)$ and 7.30-7.48 $(5 \mathrm{H}, \mathrm{m}) ; \delta_{\mathrm{C}}\left(\mathrm{CDCl}_{3}\right) 52.0(\mathrm{t}), 52.7(\mathrm{t}), 93.1(\mathrm{~d})$, 112.5 (d), 127.1 (d, 2 C), 128.8 (d, 2 C), 129.2 (d), 135.1 (s) and 140.2 (d) (Found: C, 68.9; H, 5.0; S, 18.5. Calc. for $\mathrm{C}_{11} \mathrm{H}_{12} \mathrm{O}_{2} \mathrm{~S}_{2}$ : C, $68.84 ; \mathrm{H}, 4.95 ; \mathrm{S}, 18.50 \%)$.

2-Propenyl-1,3-dithiolane 1,3-dioxide 3h. Oil (100\%), TLC (MeOH-EtOAc, 1:9) $R_{\mathrm{f}} 0.25 ; v_{\max }(\mathrm{KBr}) / \mathrm{cm}^{-1} 2967,1652$, 1395,1085 and $1027 ; m / z 179\left(\mathrm{M}^{+}+1,1 \%\right)$ and $108(100)$; $\delta_{\mathrm{H}}\left(\mathrm{CDCl}_{3}\right) 1.85(\mathrm{~d}, J 6, \mathrm{Me}), 3.50-3.86(4 \mathrm{H}, \mathrm{m}), 4.50(\mathrm{~d}, J 9$, $2-\mathrm{H}), 5.44$ (dd, $J 15$ and $\left.9,1^{\prime}-\mathrm{H}\right)$ and $6.10(1 \mathrm{H}, \mathrm{dq}, J 15$ and 6 , $2^{\prime}-\mathrm{H}$ ) (Found: $\mathrm{M}^{+}$, 178.0119. Calc. for $\mathrm{C}_{6} \mathrm{H}_{10} \mathrm{O}_{2} \mathrm{~S}_{2}: M$, 178.0122).

2-Phenyl-1,4-dithiane 1-Oxide 4.-A mixture of the dithiolane disulfoxide $3 \mathrm{a}(114 \mathrm{mg}, 0.5 \mathrm{mmol})$ and benzenethiol $\left(0.05 \mathrm{~cm}^{3}\right.$, $0.5 \mathrm{mmol})$ in $\mathrm{MeOH}\left(10 \mathrm{~cm}^{3}\right)$ was heated to $70^{\circ} \mathrm{C}$ at reflux for $36 \mathrm{~h}$. The mixture was cooled to room temperature, concentrated under reduced pressure and chromatographed on silica gel by elution with hexane-EtOAc $(1: 4)$ to give the trans- and cis-isomers of 4 in 62 and $10 \%$ yields, respectively. trans-Isomer: white solid, m.p. $180-181^{\circ} \mathrm{C}$; TLC (hexane-EtOAc, 1:4) $R_{\mathrm{f}}$ $0.16 ; v_{\max }(\mathrm{KBr}) / \mathrm{cm}^{-1} 1579,1035$ and $699 ; \mathrm{m} / z 212\left(\mathrm{M}^{+}, 34 \%\right)$ and $104(100) ; \delta_{\mathrm{H}}\left(\mathrm{CDCl}_{3}\right) 3.05-3.26(5 \mathrm{H}, \mathrm{m}), 3.60-3.70(1 \mathrm{H}$, $\mathrm{m}), 3.97(\mathrm{dd}, J 10.3$ and $2.4 .2-\mathrm{H}), 7.32-7.38(5 \mathrm{H}, \mathrm{m}) ; \delta_{\mathrm{C}^{-}}$ $\left(\mathrm{CDCl}_{3}\right) 25.5(\mathrm{t}, \mathrm{C}-5), 31.4(\mathrm{t}, \mathrm{C}-3), 52.6(\mathrm{t}, \mathrm{C}-6), 68.7(\mathrm{~d}, \mathrm{C}-2)$, 128.5 (d, 2 C), 128.75 (d), 129.1 (d, 2 C) and 135.4 (s) (Found: $\mathrm{M}^{+}, 212.0315$. Calc. for $\left.\mathrm{C}_{10} \mathrm{H}_{12} \mathrm{OS}_{2}: M, 212.0330\right)$. cis-Isomer: white solid, m.p. $135-136^{\circ} \mathrm{C}$; TLC (hexane-EtOAc, $\left.1: 4\right) R_{\mathrm{f}}$ $0.35 ; v_{\max }(\mathrm{KBr}) / \mathrm{cm}^{-1} 1597,1026$ and $696 ; \mathrm{m} / \mathrm{z} 212\left(\mathrm{M}^{+}, 65 \%\right)$ and $104(100) ; \delta_{\mathrm{H}}\left(\mathrm{CDCl}_{3}\right) 2.43(1 \mathrm{H}, \mathrm{br} \mathrm{d}, J 12.9), 2.48(1 \mathrm{H}, \mathrm{br}$ d, $J 11.8), 3.01$ (1 H, ddd, $J 14.0,12.7$ and 2.8$), 3.35(1 \mathrm{H}$, ddd, $J$ $14.0,2.8$ and 2.1$), 3.73$ ( $1 \mathrm{H}$, ddd, $J 12.9,12.7$ and 2.1$), 3.85(1 \mathrm{H}$, dd, $J 11.8$ and 2.0$), 4.02$ ( $1 \mathrm{H}$, dd, $J 11.8$ and 11.8 ) and $7.25-$ $7.38(5 \mathrm{H}, \mathrm{m}) ; \delta_{\mathrm{C}}\left(\mathrm{CDCl}_{3}\right) 16.1(\mathrm{t}, \mathrm{C}-5), 22.6(\mathrm{t}, \mathrm{C}-3), 47.8(\mathrm{t}$, C-6), 61.3 (d, C-2), 128.05 (d, 2 C), 128.6 (d), 129.0 (d, 2 C) and 135.4 (s) (Found: $\mathrm{M}^{+}, 212.0314$. Calc. for $\mathrm{C}_{10} \mathrm{H}_{12} \mathrm{OS}_{2}$ : $M, 212.0330$ ).

8,8-Dimethyl-2,5-dithiabicyclo[4.4.0]dec-9-ene 2-oxide 5.Thermolysis of the disulfoxide $3 \mathbf{b}$ by a procedure similar to that of 3a described above gave $73 \%$ yield of the bicycle 5 as a white solid, m.p. $108-109^{\circ} \mathrm{C} ; \mathrm{m} / z 216\left(\mathrm{M}^{+}, 50 \%\right)$ and $107(100)$; $\delta_{\mathrm{H}}\left(\mathrm{CDCl}_{3}\right) 1.08(\mathrm{~s}, \mathrm{Me}), 1.11(\mathrm{~s}, \mathrm{Me}), 1.74(\mathrm{br} \mathrm{d}, J 13.4,7 \beta-\mathrm{H})$, 2.04 (dd, $J 13.4$ and $13.4,7 \alpha-\mathrm{H}), 2.83$ (dd, $J 4.1$ and 4.1, 4-H), 3.02-3.18 (m, 3-H and 4-H), 3.39 (dt, $J 13.4$ and 3.2, 6-H), 3.47 (dd, $J 5.2$ and $3.2,10-\mathrm{H}), 3.60$ (dd, $J 9.2$ and $4.5,3-\mathrm{H}), 5.82$ (d, $J$ $10.0,9-\mathrm{H})$ and $6.01(\mathrm{dd}, J 10.0$ and $5.2,10-\mathrm{H}) ; \delta_{\mathrm{C}}\left(\mathrm{CDCl}_{3}\right) 21.7(\mathrm{t}$, C-4), 28.8 (q, Me), 30.3 (q, Me), 35.1 (s, C-8), 38.6 (t, C-7), 43.3 (d, C-6), 52.9 (t, C-3), 63.3(d, C-1), $120.8(\mathrm{~d}, \mathrm{C}-9)$ and $143.0(\mathrm{~d}, \mathrm{C}-$ 10) (Found: $\mathrm{M}^{+}, 216.0645$. Calc. for $\mathrm{C}_{10} \mathrm{H}_{16} \mathrm{OS}_{2}: M, 216.0642$ ).

Hept-1-en-2-yl 2-Mercaptoethyl Sulfoxide 6, Hept-1-en-2-yl 2-Phenylthioethyl Sulfoxide 7, Hept-2-en-2-yl 2-Mercaptoethyl Sulfoxide 8 and Hept-2-en-2-yl 2-Phenylthioethyl Sulfoxide 9.Compounds 6-9 were obtained by thermolysis of the disulfoxide $3 \mathrm{c}$ at $70^{\circ} \mathrm{C}$ or $110^{\circ} \mathrm{C}$ (Table 2 ) by a procedure similar to that of 3a described above. 6: Oil, TLC (hexane-EtOAc, 1:1) $\boldsymbol{R}_{\mathrm{f}}$ $0.34 ; v_{\max }($ neat $) / \mathrm{cm}^{-1} 1622$ and $1048 ; \mathrm{m} / z 206\left(\mathrm{M}^{+}, 8 \%\right), 129$ (48) and $61(100) ; \delta_{\mathrm{H}}\left(\mathrm{CDCl}_{3}\right) 0.88(\mathrm{t}, J 6.7,3, \mathrm{Me}), 1.27-1.36$ $(4 \mathrm{H}, \mathrm{m}), 1.61-1.68(2 \mathrm{H}, \mathrm{m}), 2.00-2.32(2 \mathrm{H}, \mathrm{m}), 2.69-3.02(4 \mathrm{H}$, $\mathrm{m}), 5.64(1 \mathrm{H}, \mathrm{d}, J 1.8)$ and $5.83(1 \mathrm{H}, \mathrm{d}, J 1.8) ; \delta_{\mathrm{C}}\left(\mathrm{CDCl}_{3}\right) 13.9$ $(\mathrm{q}), 17.1(\mathrm{t}), 22.3(\mathrm{t}), 27.6(\mathrm{t}), 28.4(\mathrm{t}), 31.2(\mathrm{t}), 54.9(\mathrm{t}), 116.3(\mathrm{t})$ and 152.0 (s) (Found: $\mathrm{M}^{+}$, 206.0792. Calc. for $\mathrm{C}_{9} \mathrm{H}_{18} \mathrm{OS}_{2}: M$, 206.0799). 7: Oil, TLC (hexane-EtOAc, $1: 1) R_{\mathrm{f}} 0.54 ; v_{\max }$ (neat)/ $\mathrm{cm}^{-1} 1623,1434,1052$ and $740 ; m / z(20 \mathrm{eV}) 315\left(\mathrm{M}^{+}+1,9 \%\right)$, $168(61)$ and $141(100) ; \delta_{\mathrm{H}}\left(\mathrm{CDCl}_{3}\right) 0.90(\mathrm{t}, J 6.5, \mathrm{Me}), 1.25$ $1.35(4 \mathrm{H}, \mathrm{m}), 1.40-1.60(2 \mathrm{H}, \mathrm{m}), 1.90-2.22(2 \mathrm{H}, \mathrm{m}), 2.81-3.13$ $(4 \mathrm{H}, \mathrm{m}), 5.62(1 \mathrm{H}, \mathrm{s}), 5.79(1 \mathrm{H}, \mathrm{s}), 7.21-7.37(3 \mathrm{H}, \mathrm{m})$ and 7.50 $7.56(2 \mathrm{H}, \mathrm{m}) ; \delta_{\mathrm{C}}\left(\mathrm{CDCl}_{3}\right) 13.9(\mathrm{q}), 22.3(\mathrm{t}), 27.5(\mathrm{t}), 28.35(\mathrm{t})$, $30.2(\mathrm{t}), 31.2(\mathrm{t}), 49.8(\mathrm{t}), 116.4(\mathrm{t}), 127.35(\mathrm{~d}), 128.0(\mathrm{~d}, 2 \mathrm{C}), 129.2$ (d, 2 C), $136.6(\mathrm{~s})$ and 151.8 (s) (Found: $\mathrm{M}^{+}, 314.0815$. Calc. for $\mathrm{C}_{9} \mathrm{H}_{18} \mathrm{~S}_{2}: M, 314.0832$ ). 8: Oil, TLC (hexane-EtOAc, 1:1) $R_{\mathrm{f}}$ $0.28 ; v_{\max }($ neat $) / \mathrm{cm}^{-1} 1650$ and $1048 ; \mathrm{m} / z 206\left(\mathrm{M}^{+}, 10 \%\right)$ and $103(100) ; \delta_{\mathrm{H}}\left(\mathrm{CDCl}_{3}\right) 0.89(3 \mathrm{H}, \mathrm{t}, J 6.9), 1.24-1.48(4 \mathrm{H}, \mathrm{m})$, $1.83(3 \mathrm{H}, \mathrm{s}), 2.13-2.25(2 \mathrm{H}, \mathrm{m}), 2.72-2.96(4 \mathrm{H}, \mathrm{m})$ and 6.18 $(1 \mathrm{H}, \mathrm{t}, J 7.5) ; \delta_{\mathrm{C}}\left(\mathrm{CDCl}_{3}\right) 9.2(\mathrm{q}), 13.8(\mathrm{q}), 17.5(\mathrm{t}), 22.3(\mathrm{t}), 27.8$ $(\mathrm{t}), 30.8(\mathrm{t}), 54.6(\mathrm{t}), 135.2(\mathrm{~d})$ and $136.9(\mathrm{~s})$ (Found: $\mathrm{M}^{+}$, 206.0793. Calc. for $\left.\mathrm{C}_{9} \mathrm{H}_{18} \mathrm{OS}_{2}: M, 206.0799\right)$. 9: Oil; TLC (30\% EtOAc-hexane, 3:7) $R_{\mathrm{f}} \quad 0.26 ; v_{\max }($ neat $) / \mathrm{cm}^{-1} 1574$ and 1048 ; $m / z(20 \mathrm{eV}) 315\left(\mathrm{M}^{+}+1,16 \%\right), 169(69)$ and $151(100)$; $\delta_{\mathrm{H}}\left(\mathrm{CDCl}_{3}\right) 0.90(3 \mathrm{H}, \mathrm{t}, J 7.1), 1.25-1.42(4 \mathrm{H}, \mathrm{m}), 1.77(3 \mathrm{H}, \mathrm{s})$, $2.12-2.19(2 \mathrm{H}, \mathrm{m}), 2.88-3.05(4 \mathrm{H}, \mathrm{m}), 6.12(1 \mathrm{H}, \mathrm{t}, J 7.3), 7.22-$ $7.36(3 \mathrm{H}, \mathrm{m})$ and $7.50-7.55(2 \mathrm{H}, \mathrm{m}) ; \delta_{\mathrm{C}}\left(\mathrm{CDCl}_{3}\right) 9.2(\mathrm{q}), 13.8$ $(\mathrm{q}), 22.3(\mathrm{t}), 27.7(\mathrm{t}), 30.5(\mathrm{t}), 30.7(\mathrm{t}), 49.5(\mathrm{t}), 127.3(\mathrm{~d}), 128.05$ (d, 2 C), 129.1 (d, 2 C), 135.2 (d) and 136.6 (s, 2 C) (Found: $\mathrm{M}^{+}, 314.0836$. Calc. for $\mathrm{C}_{15} \mathrm{H}_{22} \mathrm{OS}_{3}: M, 314.0832$ ).

1-Ethoxycarbonylethyl 2-Mercaptoethyl Sulfoxide 10 and 1Ethoxycarbonylethyl 2-Phenylthioethyl Sulfoxide 11.-Thermolysis of the disulfoxide $\mathbf{3 d}$ by a procedure similar to that described for compound 3a, gave the monosulfoxides $10(14 \%)$ and $11(41 \%)$. Either compound 10 or 11 existed as a mixture of diastereoisomers. 10, a mixture of two isomers $(80: 20)$ : oil, TLC (EtOAc) $R_{\mathrm{f}} 0.34 ; v_{\max }\left(\right.$ neat) $/ \mathrm{cm}^{-1} 1723$ and $1020 ; \mathrm{m} / \mathrm{z} 211$ $\left(\mathrm{M}^{+}+1,11 \%\right)$ and $150(100) ; \delta_{\mathrm{H}}\left(\mathrm{CDCl}_{3}\right) 1.32(\mathrm{t}, J 7.2, \mathrm{Me})$, 1.58 (d, J 7.3, Me major) $/ 1.53$ (d, J 7.3, minor), $2.92-3.12(4 \mathrm{H}$, $\mathrm{m}), 3.62(1 \mathrm{H}, \mathrm{q}, J 7.3) / 3.78(\mathrm{q}, J 7.3)$ and $4.25(\mathrm{q}, J 7.2$, 
$\left.\mathrm{OCH}_{2}\right) / 4.26(\mathrm{~d}, J 7.2) ; \delta_{\mathrm{C}}\left(\mathrm{CDCl}_{3}\right) 10.9(\mathrm{q}), 14.1(\mathrm{q}), 17.9(\mathrm{t})$, $55.0\left(\mathrm{t}\right.$, major)/52.6 (t, minor), $60.5(\mathrm{~d}) / 59.2(\mathrm{~d}), 62.1\left(\mathrm{t}, \mathrm{OCH}_{2}\right)$ and 168.65 (s) (Found: $\mathrm{M}^{+}, 210.0270$. Calc. for $\mathrm{C}_{7} \mathrm{H}_{12} \mathrm{O}_{3} \mathrm{~S}_{2}: M$, 210.0384). 11, a mixture of two isomers $(67: 33)$ : oil, TLC (EtOAc) $R_{\mathrm{f}} 0.53 ; v_{\max }$ (neat) $/ \mathrm{cm}^{-1} 1723,1049$ and $1045 ; \mathrm{m} / \mathrm{z}$ $319\left(\mathrm{M}^{+}+1,76 \%\right)$ and $168(100) ; \delta_{\mathrm{H}}\left(\mathrm{CDCl}_{3}\right) 1.28(\mathrm{t}, J 7.0$, Me major)/1.32 (t, $J 7.0$, Me minor), $1.52(\mathrm{~d}, J 7.3, \mathrm{Me}) / 1.59(\mathrm{~d}, J$ 7.3, Me), 3.02-3.25 (4 H, m), 3.56 (q, J 7.3)/3.64 (q, J 7.3), 4.15$4.30\left(2 \mathrm{H}, \mathrm{m}, \mathrm{OCH}_{2}\right), 7.21-7.37(3 \mathrm{H}, \mathrm{m})$ and $7.47-7.56(2 \mathrm{H}, \mathrm{m})$; $\delta_{\mathrm{C}}\left(\mathrm{CDCl}_{3}\right) 10.7(\mathrm{q}, \mathrm{Me}$ major)/10.8 (q), $14.1(\mathrm{q}), 30.8(\mathrm{t}) / 31.2$ $(\mathrm{t}), 50.1(\mathrm{t}) / 50.3(\mathrm{t}), 60.45(\mathrm{~d}), 62.0(\mathrm{t}), 127.1(\mathrm{~s}), 127.4(\mathrm{~d}), 128.1$ (d), 129 (d, 2 C) and 168.5 (s) (Found: $\mathrm{M}^{+}, 318.0442$. Calc. for $\left.\mathrm{C}_{13} \mathrm{H}_{18} \mathrm{O}_{3} \mathrm{~S}_{3}: M, 318.0418\right)$.

2-(2-Phenyl-1-phenylthioethyl)-1,3-dithiolane 1,3-Dioxide 12. - To a solution of benzenethiol $\left(0.1 \mathrm{~cm}^{3}, 1 \mathrm{mmol}\right)$ in $\mathrm{MeOH}$ $\left(2 \mathrm{~cm}^{3}\right)$ was added dropwise a solution of $\mathrm{MeMgCl}\left(0.8 \mathrm{~cm}^{3}\right.$, $1.25 \mathrm{~mol} \mathrm{dm}^{-3}$, diethyl ether). The mixture was stirred for $20 \mathrm{~min}$, after which a solution of the disulfoxide 3a $(228 \mathrm{mg}$, $1 \mathrm{mmol})$ in THF $\left(2 \mathrm{~cm}^{3}\right)$ was added to it. The mixture was stirred at room temperature for a further $24 \mathrm{~h}$ to complete the addition (TLC analysis). Saturated aqueous $\mathrm{NH}_{4} \mathrm{Cl}\left(1 \mathrm{~cm}^{3}\right)$ was then added to the mixutre and $\mathrm{MeOH}$ and THF removed by rotary evaporation. The residue was extracted with EtOAc $\left(3 \times 30 \mathrm{~cm}^{3}\right)$ and the combined extracts were dried $\left(\mathrm{Na}_{2} \mathrm{SO}_{4}\right)$ and filtered. The filtrate was concentrated and the residue chromatographed on silica gel to give the title compound $\mathbf{1 2}$ (318 $\mathrm{mg}, 91 \%$ ) containing two isomers $(50: 50)$. Isomer a was crystallised from EtOAc, m.p. $151.5-153.5^{\circ} \mathrm{C} ; v_{\max }(\mathrm{KBr}) / \mathrm{cm}^{-1}$ 2983, 1472, 1392, 1230, 1141 and 1026; $m / z 350\left(\mathrm{M}^{+}, 36 \%\right), 223$ (100) and $148(21) ; \delta_{\mathrm{H}}\left(\mathrm{CDCl}_{3}\right) 2.94-4.03(8 \mathrm{H}, \mathrm{m})$ and 7.28 $7.36(10 \mathrm{H}, \mathrm{m}) ; \delta_{\mathrm{C}}\left(\mathrm{CDCl}_{3}\right) 40.4(\mathrm{t}), 48.7(\mathrm{~d}), 51.2(\mathrm{t}), 52.1(\mathrm{t})$, 96.0 (d, C-2), 127.0, 128.2, 129.1, 129.3, 129.7, 132.8 and 136.8 (Found: $\mathrm{M}^{+}, 350.0463$. Calc. for $\mathrm{C}_{17} \mathrm{H}_{18} \mathrm{O}_{2} \mathrm{~S}_{3}: M, 350.0469$ ). Isomer $\mathbf{b}$ was isolated from the mother liquor by chromatography with EtOAc as eluent. $\delta_{\mathrm{C}}\left(\mathrm{CDCl}_{3}\right) 40.0$ (t), 47.4 (d), $50.7(\mathrm{t}), 52.2(\mathrm{t}), 97.9$ (d) $128.3,128.4,129.1,129.3,133.2,133.4$, 133.5 and 137.0 . The reaction in MeOD gave $92 \%$ yield of $\left[{ }^{2} \mathbf{H}\right]-$ 12. $\delta_{\mathrm{H}}\left(\mathrm{CDCl}_{3}\right) 3.24-4.05(7 \mathrm{H}, \mathrm{m})$ and $7.26-7.42(10 \mathrm{H}, \mathrm{m})$; $m / z 351\left(\mathrm{M}^{+}, 7 \%\right), 316(11), 242(19), 223(39), 148(40)$ and 110 (100) (Found: $\mathrm{M}^{+}, 351.0525$. Calc. for $\mathrm{C}_{17} \mathrm{H}_{17} \mathrm{DO}_{2} \mathrm{~S}_{3}: M$, $351.0531)$.

2-[1-(Dicyanomethyl)-2-phenylethyl $]-1,3-$ dithiolane 1,3-Dioxide 13.-Addition of $\mathbf{3 g}$ with malononitrile by a procedure similar to that described for the dithiolane dioxide 12 gave compound $13(42 \%)$ as a mixture of two diastereoisomers (67:23). The major isomer was crystallised from EtOAc, colourless crystals, m.p. $183-184.5^{\circ} \mathrm{C} ; v_{\max }(\mathrm{KBr}) / \mathrm{cm}^{-1} 2991$, $2252,1388,1181$ and $1021 ; m / z 306\left(\mathrm{M}^{+}, 1 \%\right), 241(99)$ and 115 (100); $\delta_{\mathrm{H}}\left(\mathrm{CDCl}_{3}\right) 3.26-3.37(3 \mathrm{H}, \mathrm{m}), 3.74-3.99(5 \mathrm{H}, \mathrm{m}), 4.38$ $\left[\mathrm{d}, J 5.1, \mathrm{CH}(\mathrm{CN})_{2}\right]$ and $7.26-7.41(5 \mathrm{H}, \mathrm{m}) ; \delta_{\mathrm{C}}\left(\mathrm{CDCl}_{3}\right) 25.6$ (d), 37.6 (t), 40.2 (d), 91.7 (d), 128.5 (d), 129.2 (d, 2 C), 129.6 (d, $2 \mathrm{C}$ ), $134.6(\mathrm{~s})$ and $167.0(\mathrm{~s}, \mathrm{CN})$ (Found: $\mathrm{M}^{+}, 306.0481$. Calc. for $\left.\mathrm{C}_{14} \mathrm{H}_{14} \mathrm{O}_{2} \mathrm{~N}_{2} \mathrm{~S}_{2}: M, 306.0496\right)$. Minor isomer: $\delta_{\mathrm{H}}\left(\mathrm{CDCl}_{3}\right)$ 3.26-3.37 (3 H, m), 3.74-3.99 $(5 \mathrm{H}, \mathrm{m}), 4.96(1 \mathrm{H}, \mathrm{d}, J 4)$ and $7.29-7.43(5 \mathrm{H}, \mathrm{m})$.

2-(1-Phenylthiopropyl)-1,3-dithiolane 1,3-Dioxide 14--Addition of benzenethiol with the dithiolane dioxide $3 \mathrm{~h}$ in a similar manner to that described for compound $\mathbf{1 2}$ gave the title compound $14(98 \%)$ containing two diastereoisomers $(57: 43)$. Two isomers were separated by HPLC on a $\mu$-Porasil column by elution with EtOAc- $\mathrm{Me}_{2} \mathrm{CO}(50: 50)$. Major isomer: white solid, m.p. $118-120^{\circ} \mathrm{C}$; HPLC (EtOAc-acetone, $\left.1: 1\right) t_{\mathrm{r}} 10.9 \mathrm{~min}$; $v_{\max }(\mathrm{KBr}) / \mathrm{cm}^{-1} 2966,1436,1393,1088$ and $1032 ; \mathrm{m} / \mathrm{z} 288$ $\left(\mathrm{M}^{+}, 73 \%\right), 212(56)$ and $180(100) ; \delta_{\mathrm{H}}\left(\mathrm{CDCl}_{3}\right) 1.26(3 \mathrm{H}, \mathrm{t}, J$ $7.2, \mathrm{Me}), 1.87-1.98,2.11-2.20(2 \mathrm{H}, \mathrm{m}), 3.42\left(\mathrm{td}, J 10,3.5,1^{\prime}-\mathrm{H}\right)$,
$3.62-3.90(5 \mathrm{H}, \mathrm{m}), 7.32-7.38$ and $7.54-7.59(5 \mathrm{H}, \mathrm{m}) ; \delta_{\mathrm{C}^{-}}$ $\left(\mathrm{CDCl}_{3}\right) 11.2(\mathrm{q}), 26.7(\mathrm{t}), 46.9(\mathrm{~d}), 50.6(\mathrm{t}), 52.1(\mathrm{t}), 97.5(\mathrm{~d})$, 128.8 (d), 129.2 (d, 2 C), 131.5 (s) and 134.5 (d, 2 C) (Found: $\mathrm{M}^{+}$, 288.0294. Calc. for $\left.\mathrm{C}_{12} \mathrm{H}_{16} \mathrm{O}_{2} \mathrm{~S}_{3}: M, 288.0312\right)$. Minor isomer: oil, HPLC (EtOAc-acetone, $1: 1) t_{\mathrm{r}} 11.4 \mathrm{~min} ; v_{\max }$ (neat)/ $\mathrm{cm}^{-1}$ 2965, 1577, 1434, 1087, 1030 (s, S=O), 750 and 692; $\delta_{\mathrm{H}}\left(\mathrm{CDCl}_{3}\right) 1.24(\mathrm{t}, J$ 7.1, Me), 1.71-1.79, 1.90-2.02 (2 H, m), $3.38\left(\mathrm{td}, J 9.1\right.$ and $\left.3.1,1^{\prime}-\mathrm{H}\right), 3.54-3.94(5 \mathrm{H}, \mathrm{m}), 7.30-7.38$ and 7.54-7.60 (5 H, m); $\delta_{\mathrm{C}}\left(\mathrm{CDCl}_{3}\right) 11.2(\mathrm{q}), 26.6(\mathrm{t}), 47.0(\mathrm{~d}), 50.4$ $(\mathrm{t}), 52.3(\mathrm{t}), 98.6(\mathrm{~d}), 128.5(\mathrm{~d}), 129.2$ (d) $131.8(\mathrm{~s})$ and 134.1 (d, 2 C) (Found: $\mathrm{M}^{+}, 288.0302$. Calc. for $\mathrm{C}_{12} \mathrm{H}_{16} \mathrm{O}_{2} \mathrm{~S}_{3}: M$, 288.0312).

2-[1-(Dicyanomethyl)]propyl-1,3-dithiolane 1,3-Dioxide15.Method A: Addition of the dithiolane dioxide $3 \mathrm{~h}$ with malononitrile by a procedure similar to that described for compound 12 gave the title compound $15(46 \%)$ containing two diastereoisomers (67:33). Method B: Neutral alumina (0.5 g) was activated by heating at $140^{\circ} \mathrm{C}$ for $2 \mathrm{~h}$, cooled and added to a solution of malononitrile $(33 \mathrm{mg}, 0.5 \mathrm{mmol})$ in THF $\left(1 \mathrm{~cm}^{3}\right)$. The mixture was stirred for $0.5 \mathrm{~h}$ after which a solution of the dithiolane dioxide $3 \mathrm{~h}(89 \mathrm{mg}, 0.5 \mathrm{mmol})$ in THF $\left(1 \mathrm{~cm}^{3}\right)$ was added to it and the whole stirred for $72 \mathrm{~h}$ under an atmosphere of argon. After removal of the THF, the residue was extracted with EtOAc $\left(2 \times 10 \mathrm{~cm}^{3}\right)$. The combined extracts were washed with brine, dried $\left(\mathrm{Na}_{2} \mathrm{SO}_{4}\right)$ and filtered. The filtrate was concentrated and the residue chromatographed on silica gel by elution with EtOAc to give the title compound 15 (34 mg, 28\%) as a mixture of two isomers (56:44); colourless solid, m.p. 125$127^{\circ} \mathrm{C}$; TLC (EtOAc) $R_{\mathrm{f}} 0.25 ; v_{\max }(\mathrm{KBr}) / \mathrm{cm}^{-1} 2971,2168$ $(\mathrm{CN}), 1628,1094$ and 1026; $m / z 244\left(\mathrm{M}^{+}, 4 \%\right)$ and $108(100)$; $\delta_{\mathrm{H}}\left(\mathrm{CDCl}_{3} / \mathrm{CD}_{3} \mathrm{OD}\right) 1.21(\mathrm{t}, J 7.4, \mathrm{Me}), 2.05-2.19(2 \mathrm{H}, \mathrm{m}$, $\left.2^{\prime}-\mathrm{H}\right), 2.81-2.92\left(1 \mathrm{H}, \mathrm{m}, 1^{\prime}-\mathrm{H}\right), 3.67-3.90(5 \mathrm{H}, \mathrm{m}), 4.49$ (d, J 5.8, $\mathrm{CH}(\mathrm{CN})_{2}$ major)/4.99 (d, J 3.0, minor) (Found: $\mathrm{M}^{+}, 244.0341$. Calc. for $\mathrm{C}_{9} \mathrm{H}_{12} \mathrm{~N}_{2} \mathrm{O}_{2} \mathrm{~S}_{2}: M, 244.0339$ ). When the reaction was conducted in MeOD, $\left[{ }^{2} \mathrm{H}\right]-15$ was obtained, accompanied by recovery of the starting material $3 \mathrm{~h} ; m / z 245\left(\mathrm{M}^{+}, 1 \%\right)$ and 108 $(100) ; \delta_{\mathrm{H}}\left(\mathrm{CDCl}_{3} / \mathrm{CD}_{3} \mathrm{OD}\right) 1.28(\mathrm{~d}, J$ 7.2, Me), 2.18-2.28 (1 H, $\left.\mathrm{m}, 2^{\prime}-\mathrm{H}\right), 2.89-3.01\left(1 \mathrm{H}, \mathrm{m}, 1^{\prime}-\mathrm{H}\right), 3.74-3.95(5 \mathrm{H}, \mathrm{m}), 4.54$ [d, $J$ $5.9, \mathrm{CH}(\mathrm{CN})_{2}$, major $]$ and $5.07\left(\mathrm{~d}, J 3.0\right.$, minor) (Found: $\mathrm{M}^{+}$, 245.0401. Calc. for $\left.\mathrm{C}_{9} \mathrm{H}_{11} \mathrm{DN}_{2} \mathrm{O}_{2} \mathrm{~S}_{2}: M, 245.0402\right)$.

2-\{1-[Bis(ethoxycarbonyl)methyl] $\}$ propyl-1,3-dithiolane 1,3Dioxide 16. - Addition of the dithiolane dioxide $3 \mathrm{~h}$ with diethyl malonate by a procedure similar to that described for compound 12 gave the title compound $16(46 \%)$ containing two diastereomers (71:29). Liquid, TLC (MeOH-EtOAc, 1:9) $R_{\mathrm{f}}$ $0.4 ; v_{\max }($ neat $) / \mathrm{cm}^{-1} 2975,1722(\mathrm{~s}, \mathrm{C}=\mathrm{O}), 1231,1093$ and 1032; $m / z 339\left(\mathrm{M}^{+}+1,2 \%\right)$ and $108(100) ; \delta_{\mathrm{H}}\left(\mathrm{CDCl}_{3}\right) 1.02(\mathrm{t}, J$ 7.3, Me major)/1.05 (t, $J$ 7.3, Me minor), $1.27-1.37(6 \mathrm{H}, \mathrm{m})$, 1.54-1.74 (2 H, m), 2.85-2.94 (1 H, m), 3.50-3.98 (7 H, m) and 4.19-4.39 (3 H, m) (Found: $\mathrm{M}^{+}, 338.0855$. Calc. for $\left.\mathrm{C}_{13} \mathrm{H}_{22} \mathrm{O}_{6} \mathrm{~S}_{2}: M, 338.0858\right)$.

2-\{1-[(4,5-Dihydro-1,3-thiazol-2-yl)thio $]\}$ propyl-1,3-dithiolane 1,3-Dioxide 17.--Addition reaction of the dithiolane dioxide $3 \mathrm{~h}$ with 2 -mercapto-4,5-dihydrothiazole by a procedure similar to that described for compound 12 gave the title compound $17(83 \%)$. Liquid, TLC (MeOH-EtOAc, 1:9); $R_{\mathrm{f}}$ $0.1 ; v_{\max }$ (neat) $/ \mathrm{cm}^{-1} 1625$ and $1030 ; m / z 297\left(\mathrm{M}^{+}, 1 \%\right)$ and 119 $(100) ; \delta_{\mathrm{H}}\left(\mathrm{CDCl}_{3}\right), 1.03(\mathrm{t}, J 7.2, \mathrm{Me}), 1.82-1.94(1 \mathrm{H}, \mathrm{m}), 2.02-$ $2.15(1 \mathrm{H}, \mathrm{m}), 3.35-3.49(2 \mathrm{H}, \mathrm{m}), 3.60-3.99(6 \mathrm{H}, \mathrm{m})$ and 4.11 $4.22(2 \mathrm{H}, \mathrm{m}) ; \delta_{\mathrm{C}}\left(\mathrm{CDCl}_{3}\right) 10.2(\mathrm{q}), 25.7(\mathrm{t}), 28.0(\mathrm{t}), 51.2(\mathrm{t}), 53.0$ $(\mathrm{t}), 53.4(\mathrm{t}), 53.6(\mathrm{t}), 94.9(\mathrm{~d}, \mathrm{C}-2)$ and $199.4(\mathrm{~s})$ (Found: $\mathrm{M}^{+}$, 296.9968. Calc. for $\left.\mathrm{C}_{9} \mathrm{H}_{15} \mathrm{NO}_{2} \mathrm{~S}_{4}: M, 296.9986\right)$.

2-Propylidene-1,3-dithiolane 1,3-Dioxide 18.-By a similar procedure to that described above for the dithiolane dioxide $\mathbf{3 a}$, 
2-propylidene-1,3-dithiolane ${ }^{20}$ was oxidized with $\mathrm{NaIO}_{4}(1$ equiv.) to give the 2-propylidene-1,3-dithiolane 1-oxide 18a $(59 \%)$ as a mixture of $E$ - and $Z$-isomers (79:21); pale yellow oil, TLC (EtOAc-hexane, 1:1) $R_{\mathrm{f}} \quad 0.13 ; v_{\max }$ (neat) $/ \mathrm{cm}^{-1} \quad 2965$, $1676,1397,1171$ and $1045 ; m / z 162\left(\mathrm{M}^{+}, 47 \%\right)$ and $145(100)$; $\delta_{\mathrm{H}}\left(\mathrm{CDCl}_{3}\right) 1.06(\mathrm{t}, J 7.5, \mathrm{Me}), 2.12-2.27(2 \mathrm{H}, \mathrm{m}), 2.57-2.78$, $3.40-3.64,3.98-4.12(4 \mathrm{H}, \mathrm{m})$ and $6.46\left(\mathrm{t}, J 7.1,1^{\prime}-\mathrm{H} E-\right.$ isomer) $/ 6.09\left(\mathrm{t}, J 7.8,1^{\prime}-\mathrm{H} Z\right.$-isomer); $\delta_{\mathrm{C}}\left(\mathrm{CDCl}_{3}\right) 12.4\left(\mathrm{q}, \mathrm{C}-3^{\prime}\right.$ $E$-isomer)/14.2 (q, C-3' Z-isomer), $25.6\left(\mathrm{t}, \mathrm{C}-2^{\prime}\right) / 26.5(\mathrm{t}), 31.9(\mathrm{t}$, C-4)/31.4 (t), $54.9(\mathrm{t}, \mathrm{C}-5) / 55.6(\mathrm{t}), 135.3\left(\mathrm{~d}, \mathrm{C}-1^{\prime}\right) / 134.6(\mathrm{~d})$ and 145.5 (s, C-2)/144.5 (s) (Found: $\mathrm{M}^{+}, 162.0176$. Calc. for $\mathrm{C}_{6} \mathrm{H}_{10} \mathrm{OS}_{2}: M, 162.0173$ ). Further oxidation of the monosulfoxide 18a with $m$-CPBA ( 1 equiv.) gave $42 \%$ yield of the dioxide 18: oil; $v_{\max }$ (neat) $/ \mathrm{cm}^{-1}$ 2973, 1607, 1459, 1327 and $1092 ; \mathrm{m} / z 178\left(\mathrm{M}^{+}, 24 \%\right)$ and $135(100) ; \delta_{\mathrm{H}}\left(\mathrm{CDCl}_{3}\right) 1.17(\mathrm{t}, J$ $7.5, \mathrm{Me}), 2.69(2 \mathrm{H}, \mathrm{qd}, J 7.7$ and 7.5$), 3.58-3.86(4 \mathrm{H}, \mathrm{m})$ and $7.27\left(\mathrm{t}, J 7.7,1^{\prime}-\mathrm{H}\right) ; \delta_{\mathrm{C}}\left(\mathrm{CDCl}_{3}\right) 12.5(\mathrm{q}), 26.5(\mathrm{t}), 50.1(\mathrm{t}), 50.4$ (t), $155.7(\mathrm{~s})$ and 156.7 (d) (Found: $\mathbf{M}^{+}, 178.0115$. Calc. for $\left.\mathrm{C}_{6} \mathrm{H}_{10} \mathrm{O}_{2} \mathrm{~S}_{2}: M, 178.0122\right)$.

2-(2-Methoxypropylidene)-1,3-dithiolane 1-Oxide 19.--A methanolic solution of equivalent amounts of the dithiolane dioxide $3 \mathrm{~h}$, acetic acid and $\mathrm{MeMgCl}\left(1.25 \mathrm{~mol} \mathrm{dm}^{-3}\right.$, diethyl ether $)$ were stirred at room temperature for $1 \mathrm{~h}$ to give the title compound $19(55 \%)$, containing four diastereoisomers $(\mathbf{a}, \mathbf{b}, \mathbf{c}$ and d, $33: 33: 17: 17)$. Isomers $\mathbf{a}$ and $\mathbf{b}$ had $Z$-configuration whereas c and d had E-configuration by analysis of the ${ }^{1} \mathbf{H}$ NMR spectrum. Oil, TLC (MeOH-EtOAc, $1: 9) R_{\mathrm{f}} 0.5 ; v_{\max }$ (neat)/ $\mathrm{cm}^{-1} 2973,1594,1444,1084$ and 1042; $\mathrm{m} / z 192\left(\mathrm{M}^{+}, 7 \%\right)$ and $175(86) ; \delta_{\mathrm{H}}\left(\mathrm{CDCl}_{3}\right) 1.30(\mathrm{~d}, J 6.5, E$-isomer) $/ 1.315(\mathrm{~d}, J 6.3$, $Z$-isomer) $/ 1.32$ (d, $J 6.6, E$-isomer) $/ 1.38$ (d, $J 6.2, Z$-isomer), 2.75 $(1 \mathrm{H}, \mathrm{m}), 3.28$ (s, OMe $Z$-isomer)/3.31 (s, $E$-isomer) $/ 3.36$ (s, $E$ isomer) $/ 3.37(\mathrm{~s}, Z$-isomer), $3.49-3.86(2 \mathrm{H}, \mathrm{m}), 3.90-4.13(\mathrm{~m}, Z$ isomer) $/ 4.444 .57\left(\mathrm{~m}, E\right.$-isomer) and $5.94\left(\mathrm{~d}, J 8.7,1^{\prime}-\mathrm{H}, Z\right.$ isomer) $/ 5.99$ (d, $J 8.7, Z$-isomer) $/ 6.40$ (d, $J 5.0, E$-isomer) $/ 6.44$ (d, $J$ 5.0, $E$-isomer) (Found: $\mathrm{M}^{+}, 192.0271$. Calc. for $\left.\mathrm{C}_{7} \mathrm{H}_{12} \mathrm{O}_{2} \mathrm{~S}_{2}: M, 192.0278\right)$.

1-Allyl-2-methylpent-4-enylidene-1,3-dithiolane 20.--Under an atmosphere of argon, trifluoroacetic anhydride $\left(0.15 \mathrm{~cm}^{3}\right.$, $1 \mathrm{mmol}$ ) was added dropwise to a solution of the dithiolane dioxide $3 \mathrm{~h}(89 \mathrm{mg}, 0.5 \mathrm{mmol})$ in $\mathrm{CH}_{2} \mathrm{Cl}_{2}\left(2 \mathrm{~cm}^{3}\right)$ at $0^{\circ} \mathrm{C}$. The reddish mixture was stirred for $15 \mathrm{~min}$ and then allyltrimethylsilane $\left(0.16 \mathrm{~cm}^{3}, 1 \mathrm{mmol}\right)$ was added to it and the mixture stirred at room temperature for $12 \mathrm{~h}$. After dilution with water $\left(2 \mathrm{~cm}^{3}\right)$ the mixture was concentrated then extracted with EtOAc $\left(2 \times 10 \mathrm{~cm}^{3}\right)$. The combined extracts were washed with brine, dried $\left(\mathrm{Na}_{2} \mathrm{SO}_{4}\right)$ and filtered. The filtrate was concentrated and the residue chromatographed on silica gel by elution with EtOAc-hexane $(2: 98)$ to give the title compound $20(60 \mathrm{mg}$, $53 \%$ ). Oil, TLC (EtOAc-hexane, 2:98) $R_{\mathrm{f}} 0.35 ; v_{\max }$ (neat)/ $\mathrm{cm}^{-1} 1673 ; m / z 226\left(\mathrm{M}^{+}, 11 \%\right)$ and $185(100) ; \delta_{\mathrm{H}}\left(\mathrm{CDCl}_{3}\right) 1.03$ (3 H, d, J 6.9), 2.02-2.27 (2 H, m), 2.61-2.78 (1 H, m), 2.86-2.91 $(2 \mathrm{H}, \mathrm{m}), 2.32(4 \mathrm{H}, \mathrm{s}$, dithiolane ring $\mathrm{H}), 4.91-5.14(4 \mathrm{H}, \mathrm{m})$ and 5.63-5.86 (2 H, m); $\delta_{\mathrm{C}}\left(\mathrm{CDCl}_{3}\right) 18.25(\mathrm{q}, \mathrm{Me}), 36.8(\mathrm{t}), 37.4(\mathrm{t}$, dithiolane ring $\mathrm{C}), 39.45(\mathrm{t}), 42.3(\mathrm{~d}), 115.6(\mathrm{t}, 2 \mathrm{C}), 128.5(\mathrm{~s})$, $131.0(\mathrm{~s}), 135.5$ (d) and 137.25 (d) (Found: $\mathrm{M}^{+}, 226.0853$. Calc. for $\left.\mathrm{C}_{12} \mathrm{H}_{18} \mathrm{~S}_{2}: M, 226.0850\right)$.

\section{Acknowledgements}

We are grateful to the National Science Council for financial support (Grant NSC82-0208-M002-31).

\section{References}

1 T. Durst, in Comprehensive Organic Chemistry, eds. D. Barton and W. D. Ollis, Pergamon Press, 1979, vol. 3, pp. 121-170; eds. S. Patai, Z. Rappoport and C. Sterling, The Chemistry of Sulphones and Sulphoxides, Wiley, 1988.

2 G. Solladie, in Asymmetric Synthesis, ed. J. D. Morrison, Academic Press, 1983, vol. 2, p. 157; G. H. Posner, Asymmetric Synthesis, ed. J. D. Morrison, Academic Press, 1983, vol. 2, p. 225.

3 P. C. B. Page and E. S. Namwindwa, Synlett., 1991, 80 and references cited therein; V. K. Aggarwal, M. Lightowler and S. D. Lindell, Synlett., 1992, 730 and references cited therein; N, Khiar, I. Fernández and F. Alcuda, Tetrahedron Lett., 1993, 34, 123.

4 V. K. Aggarwal, I. W. Davies, J. Maddock, M. F. Mahon and K. C. Molloy, J. Chem. Soc., Perkin Trans. 1, 1992, 662.

5 F. A. Carey, O. D. Dailey Jr. and T. E. Fromuth, Phosphorus Sulfur, 1981, 10, 163.

6 O. Samuel, B. Ronan and H. B. Kagan, J. Organomet. Chem., 1989, 370, 43 .

7 J.-M. Fang, W.-C. Chou, G.-H. Lee and S.-M. Peng, J. Org. Chem., $1990,55,5515$ and the references cited therein.

8 O. Bortolini, F. D. Furia, G. Licini and M. Rossi, Tetrahedron Lett., 1986, 27, 6257.

9 F. A. Carey, O. D. Dailey Jr. and W. C. Hutton, J. Org. Chem., 1978, 43, 96.

10 C. H. Chen, Tetrahedron Lett., 1976, 25; N. Ueda, H. Shimizu, T. Kataoka and M. Hori, Tetrahedron Lett., 1984, 25, 757; W. S. Lee, K. Lee, K. D. Nam and Y. J. Kim, Tetrahedron, 1991, 47, 8091.

11 D. R. Hogg, in Comprehensive Organic Chemistry, eds. D. Barton and W. D. Ollis, Pergamon Press, 1979, vol. 3, pp. 264-265.

12 G. H. Posner and P. W. Tang, J. Org. Chem., 1978, 43, 4131.

13 S. Oae and Y. Uchida, Acc. Chem. Res., 1991, 24, 202.

14 D. A. Evans and G. C. Andrews, Acc. Chem. Res., 1974, 7, 147; F. A. Carey, P. M. Smith, R. J. Maher and R. F. Bryan, J. Org. Chem., 1977, 42, 961 .

15 B. C. Ranu, S. Bhar and D. C. Sarkar, Tetrahedron Lett., 1991, 32, 2811.

16 F. A. Carey and O. D. Dailey Jr., Phosphorus Sulfur, 1981, 10, 169; E. Schaumann, in Perspectives in Organosulfur Chemistry, eds. B. Zwanenburg and A. Klunder, Elsevier, 1986, p. 251.

17 D. Pandy-Szekeres, G. Déléris, J.-P. Picard, J.-P. Pillot and R. Calas, Tetrahedron Lett., 1980, 21, 4267; R. Hunter and C. D. Simon, Tetrahedron Lett., 1986, 27, 1385; I. Mori, P. A. Bartlett and C. H. Heathcock, J. Org. Chem., 1990, 55, 5966; W.-L. Cheng and T.-Y. Luh, J. Org. Chem., 1992, 57, 3516; W. H. Chan, A. W. M. Lee and E. T. T. Chan, J. Chem. Soc., Perkin Trans. 1, 1992, 945.

18 C.-C. Yang and J.-M. Fang, J. Chem. Soc., Perkin Trans. 1, 1992 , 3085.

19 A. Hoppmann, P. Weyerstahl and P. Zummack, Justus Liebigs Ann. Chem., 1977, 1547; J.-M. Fang, L.-F. Liao and B.-C. Hong, J. Org. Chem., 1986, 51, 2828.

20 E. J. Corey and A. P. Kozikowski, Tetrahedron Lett., 1975, 11, 925.
Paper 3/04409B

Received 26th July 1993 Accepted 6th October 1993 